\title{
Terahertz Radiation Generation Process In The Medium Based On The Array of The Elongated Nanoparticles
}

\section{Nail Sadykov ( $\sim$ n.r.sadykov@rambler.ru )}

National Research Nuclear University "MEPhl" https://orcid.org/0000-0003-1381-249X Irina Alekseevna Pilipenko

National Research Nuclear University MEPhl: Nacional'nyj issledovatel'skij adernyj universitet Moskovskij inzenerno-fiziceskij institut

\section{Semyon Evgenievich Jolnirov}

National Research Nuclear University MEPhl: Nacional'nyj issledovatel'skij adernyj universitet Moskovskij inzenerno-fiziceskij institut

\section{Research Article}

Keywords: radiation, nanoribbons, metallic, multicascade, terahertz

Posted Date: August 4th, 2021

DOI: https://doi.org/10.21203/rs.3.rs-684447/v1

License: (c) (i) This work is licensed under a Creative Commons Attribution 4.0 International License. Read Full License

Version of Record: A version of this preprint was published at Optical and Quantum Electronics on December 7th, 2021. See the published version at https://doi.org/10.1007/s11082-021-03386-w. 


\title{
Terahertz radiation generation process in the medium based on the array of
}

\section{the elongated nanoparticles}

\author{
N.R. Sadykov , I.A. Pilipenko, S.E. Jolnirov \\ Department of Physics, National Research Nuclear University MEPhI (Moscow Engineering \\ Physics Institute), Kashirskoe shosse, 31, Moscow, 115409, Russian Federation
}

\begin{abstract}
We conduct a theoretical study and numerical simulations of terahertz radiation generation in the medium based on armchair-edge nanoribbons and zigzag nanotubes with metallic conductivity. The multicascade mechanism of radiation generation is considered in the task of terahertz radiation generation. The level of the injection current in nanoparticle arrays has been estimated. The task expands to the similar medium where radiation current is generated with the use of infrared radiation stimulated absorption, for example, radiation of a $\mathrm{CO}_{2}$ laser. Numerical results for the effective dielectric function have been obtained with the use of the effective mediumapproximation, the Maxwell-Garnett's theory and the Maxwell-Garnett model with the ClausiusMossotti correction (geometrical model arrays).
\end{abstract}

\section{Introduction}

A quantum cascade laser (QCL) is a device, which combines aspects from different fields such as nanoelectronics and quantum engineering, plasmonics, as well as subwavelength and nonlinear photonics. Since their invention, QCLs [1] have been rapidly developed into an important class of lasers, covering a wide wavelength range $3-300 \mu \mathrm{m}$.

Such a light amplification is possible in intersubband transitions, i.e. transitions between quantised energy states within one energy band of a semiconductor, which was first predicted by [2] in a seminal paper. We agree that the fast nonradiative longitudinal optical (LO) phonon emission rate can be harnessed for the laser process (the first QCL intersubbaned laser, designed to emit at $4.3 \mu \mathrm{m}$ wavelength, was demonstrated in [3, 4]), rather than being an hindrance for intersubbaned laser action. Zheng et al. [5, Zhang] considered, that infrared lasers had been developed on the basis of and intersubband transition in semiconductor heterostructures with quantum wells and super lattices of type-II. After appearance of works [3, 4], based on the work [2], the significant progress in the pulse lasers development is reached.

For instance, a Continuous Wave laser, functioning at cryogenic temperatures was invented [6, 7]; a laser, functioning in a pulsed mode up to room temperature was also invented by Faist et al. [8]. Considerable progress in the power output [9] and the available frequency range [10, 11] was made. The first far-infrared QS lasers with wavelengths larger than $20 \mu \mathrm{m}$ (21.5 and $24 \mu \mathrm{m})$ 
were shown by Colombelli et al. [12]. Generation of ultra-short pulses in mid-infrared [13] and terahertz QCLs $[14,15]$ is a promising direction in Physics.

Development of innovative QCL types and subsequent design optimization, systematic improvements of the quantum cascade lasers operating temperature, efficiency and a spectral range require detailed simulation of the underlying physical processes in these structures [16]. For instance, $\mathrm{THz}$ generation at the room temperature was obtained at the power levels up to $120 \mu \mathrm{W}$ $[17,18]$. It is necessary for most technical applications to push the available room temperature output power to a few $\mathrm{mW}$. Simulation of such terahertz QCL sources is especially demanding, because it requires the coupled simulation of two mid-infrared QCLs and careful simulations of the nonlinear susceptibility and the associated frequency conversion process in the heterostructure [19]. Thus, a quantitative simulation, reliable numerical design optimization and explorations must also include the optical cavity [20, 21, 18, 22]. A further example is surface emission schemes based on one- and two-dimensional photonic crystal structures, [23, 24, 25] offering tailorable emission properties and improved beam quality. The simulation of such subwavelength-structured cavities requires advanced electromagnetic simulation, e.g., based on the coupled mode theory [26] or even full finite-difference time-domain simulations of Maxwell's equations [25, 27].

We can see the unique situation in the case of small-sized nanostructures. Evolution equations, written in such structures, represent the difference approximation of differential equations. Studying the processes of Low-dimensional nanostructures permits the application of well-established numerical methods and properties of different schemes. For example, the evolution of charge carriers (electrons and holes) in the terms of a tight binding model for twoatomic unit cells is described by the Dirac equation as particles and antiparticles with zero-mass $[28,29,30$.$] . One of the prominent results is the usage of nanotubes of the continuum model (kp-$ type model) for description of charge carries in graphene [31, 32]. In [33], based on the kp-type model, an approach for the approximate calculation of the characteristics of electromagnetic guided waves on almost circular, closely packed bundles of parallel, identical, and metallic carbon nanotubes is proposed. A kp-type model yields the polarizability scalar and the antenna efficiency of a finite-length SWCNT bundle in the long-wavelength regime over a wide frequency range spanning the terahertz and the near-infrared regimes. The approach used in [33] is similar to the approach from [34], in which the Fraunhofer's multiple slits diffraction model is used to explain the far-field pattern of a phase-locked quantum cascade laser array. In [35] the author's approach combines the tight-binding model with the continuum model of kp-type to investigate finite length graphene nanoribbon with armchair edges subjected to the external electric field longitudinal to the edges and derive approximate solutions for the electron energies and wave functions around the Dirac point. In the work on the simulation of a cascade laser similar to [28, 36] it is shown that generation of terahertz radiation is possible at reasonable experimental parameters. It is also discussed how generation of radiation by Cherenkov mechanism is possible [37]. In addition it is claimed that equations obtained for electrons in armchair graphene nanoribbons can be reduced to the Majorana equation [38].

The paper focuses on a theoretical study and numerical simulation of the task of amplification of terahertz radiation by analogy with QC lasers in medium, based on non-interacting armchair edge nanoribbons with metallic conductivity, and on the estimation of the value of generating radiation and the value of radiative current density. The case of noninteracting metallic nanotubes for non-densely and randomly distributed composites of particles is discussed in [36]. The effective dielectric function in the geometrical model arrays is defined by the individual particle polarizability with consideration to the geometry of the array (geometric factor). It is 
shown that both the medium based on randomly distributed composites of particles (the effective medium-approximation, the Maxwell-Garnett's Theory [MGT]) and the array of particles (the arrays geometrical model) from parallel oriented CNTs and armchair-edge nanoribbons.

The structure of the paper is the following. Section 1 is an introduction that presents the literature review. Section 2 presents the interaction of a classical optical field with an equidistant multilevel system using the density matrix formalism. The wave functions, the eigenvalues of the energy states of charge carriers, and the matrix elements of the dipole moments in armchair nanoribbons and zigzag nanotubes of finite length are written out. In Section 3, in the medium based on the array of noninteracting zigzag nanotubes or noninteracting armchair-edge nanoribbons, the process of generating terahertz radiation is considered on the basis of the obtained differential-difference equation for the diagonal elements of the density matrix. Section 4 is devoted to a theoretical study of the permittivity function for a crystal lattice of scattering nanoparticles with a metallic type of conduction (CNTs and GNLs) with nanoparticle centers located at the same distance on the basis of the mean field theory and the Maxwell - Garnett approach. Section 5 provides the results of numerical simulation of the task of terahertz radiation generation in the medium based on the parallel oriented graphene armchair edge nanoribbons and zigzag nanotubes. Section 5 also presents the calculation results obtained for the effective dielectric functions in medium based on the array of non-interacting zigzag nanotubes or noninteracting nanoribbons with the edges of the chair. Section 6 is devoted to the discussion of obtained results. Conclusion is the last section.

\section{The equation for density matrix elements in the array of nanoparticles}

\section{2a. Nanotubes}

First we consider the array of chiral nanotubes as the nonlinear media and then generalise the obtained results for the array of armchair-edge nanoribbons. The theoretical part of the task for the zigzag nanotubes is viewed in [36], in which the analytically obtained results in [39] are used. In [39] on account of an elementary phase cell, which contains sublattice A and sublattice B, the wave functions, equidistant energy levels and dipole matrix elements of laser transition in the presence of a longitudinal electric field are analytically obtained. In [40] as distinct from [39] the array of chiral nanotubes is viewed. We write the Liouville equation for the considered multilevel system for the density matrix

$$
i \mathrm{~h} \partial \hat{\rho} / \partial t=[\hat{H}, \hat{\rho}]
$$

Where $[\hat{H}, \hat{\rho}]$ is the commutator of Hamilton operator $\hat{H}$. We use equation (2.1) (where $T_{1}$ is the longitudinal time relaxation; $T_{2}$ is the transverse relaxation time) and then write the interaction of a classical optical field with a multilevel system with the use of density matrix formalism as) $[36,35]$ 


$$
\begin{aligned}
& \frac{\partial \rho_{k, k-1}}{\partial t}+\frac{\rho_{k, k-1}}{T_{2}}=-i \Omega \rho_{k, k-1}-i \frac{\mu}{\mathrm{h}}\left(\rho_{k k}-\rho_{k-1, k-1}\right) \frac{\bar{E}}{2} \\
& -i \frac{\mu}{\mathrm{h}}\left(\rho_{k+1, k-1}-\rho_{k, k-2}\right) \frac{\bar{E}^{*}}{2}, \quad k=2, \ldots, K, \\
& \frac{\partial \rho_{k+1, k-1}}{\partial t}+\frac{\rho_{k+1, k-1}}{T_{2}}=-i 2 \Omega \rho_{k+1, k-1}-i \frac{\mu}{\mathrm{h}}\left(\rho_{k+1, k}-\rho_{k, k-1}\right) \frac{\bar{E}}{2} \\
& -i \alpha \frac{\mu}{\mathrm{h}}\left(\rho_{k+2, k-1}-\rho_{k+1, k-2}\right) \frac{\bar{E}^{*}}{2}, \\
& \frac{\partial \rho_{k k}}{\partial t}+\frac{\rho_{k k}}{T_{1}}=i \frac{\mu}{\mathrm{h}}\left(\rho_{k+1, k}-\rho_{k, k-1}\right) \frac{\bar{E}^{*}}{2}+\text { c. c., } \quad k=2, \ldots, K-1, \\
& \frac{\partial \rho_{K K}}{\partial t}+\frac{\rho_{\mathrm{K}, \mathrm{K}}}{T_{1}}=-i \frac{\mu}{\mathrm{h}} \rho_{K, K-1} \frac{\bar{E}^{*}}{2}+\text { c.c., } \quad \frac{\partial \rho_{11}}{\partial t}+\frac{\rho_{11}}{T_{1}}=i \frac{\mu}{\mathrm{h}} \rho_{21} \frac{\bar{E}^{*}}{2}+\text { c.c., }
\end{aligned}
$$

where $\hat{H}=-\hat{d}_{z} E_{z}$ is the Hamilton operator; $E_{z}=\bar{E} / 2+\bar{E}^{*} / 2, \bar{E}$ and $\bar{E}^{*}$ are the complex conjugate quantities; $\bar{E} \sim \exp (-i \omega t),\left(d_{z}\right)_{f n}=e(z)_{f n}=-2 L /\left[\pi^{2}(f-n)^{2}\right]$ are the dipole matrix element of laser transition), $\quad \mu=\left(d_{z}\right)_{k, k-1}=e(z)_{k, k-1}$, $\left(d_{z}\right)_{k+1, k-2}=\left(d_{z}\right)_{k+2, k-1}=\mu / 9, \quad \bar{\varepsilon}_{k}=\sqrt{3} \pi\left|\gamma_{0}\right| a(1+2 k) /(4 L), \Omega=\left(\bar{\varepsilon}_{k}-\bar{\varepsilon}_{k-1}\right) / \mathrm{h}$ $=\sqrt{3} \pi \gamma_{0} a /(2 \mathrm{~h} L), \gamma_{0}=2.7 \mathrm{eV}$ are the hopping integral. From (2.2) the time dependence of the density matrix elements of the density matrix follows

$$
\begin{aligned}
& \rho_{k, k-1}=\rho_{k-1, k}^{*} \sim \exp (-i \omega t), \quad \rho_{k+1, k-1}=\rho_{k-1, k+1}^{*} \sim \exp (-2 i \omega t), \\
& \rho_{k+2, k-1}=\rho_{k-1, k+2}^{*} \sim \exp (-3 i \omega t) .
\end{aligned}
$$

We transform quantity $\rho_{k+1, k-1}$ analytically. From Eq. (2.2) one can obtain

$$
\rho_{k+1, k-1}=\frac{\mu \bar{E}\left(\rho_{k+1, k}-\rho_{k, k-1}\right)}{2 \mathrm{~h}\left[2(\omega-\Omega)+i \mathrm{~T}_{2}^{-1}\right]}+\frac{\alpha \mu \bar{E}^{*}\left(\rho_{k+2, k-1}-\rho_{k+1, k-2}\right)}{2 \mathrm{~h}\left[2(\omega-\Omega)+i \mathrm{~T}_{2}^{-1}\right]} .
$$

If we substitute quantity $\rho_{k+1, k-1}$ from (2.4) in the first Eq. (2.2), the summands will appear in the right part of the equation

$$
\sim \alpha^{2}|\mu \bar{E}|^{2}\left|\partial \rho_{k, k-1} / \partial k\right| /\left(\mathrm{h}^{2} T_{2}^{-1}\right) \sim \alpha^{2}|\mu \bar{E}|^{2}\left|\rho_{k, k-1}\right| /\left(\mathrm{h}^{2} T_{2}^{-1} K\right)<<\Omega\left|\rho_{k, k-1}\right| .
$$


For the same reason we neglect the second summand in Eq. (2.4). As a result, Eq. (2.2) for the density matrix elements transforms into

$$
\begin{aligned}
& \frac{\partial \rho_{k, k-1}}{\partial t}+\frac{\rho_{k, k-1}}{T_{2}}=-i \Omega \rho_{k, k-1}-i \frac{\mu}{\mathrm{h}}\left(\rho_{k k}-\rho_{k-1, k-1}\right) \frac{\bar{E}}{2}, \quad k=2, \ldots, K, \\
& \frac{\partial \rho_{k k}}{\partial t}+\frac{\rho_{k k}-\rho_{k k}^{(0)}}{T_{1}}=\left[i \frac{\mu}{\mathrm{h}}\left(\rho_{k+1, k}-\rho_{k, k-1}\right) \frac{\bar{E}^{*}}{2}+\text { c. c. }\right] \\
& +\frac{J_{k}(V, T)}{e}, \quad k=2, \ldots, K-1, \\
& \frac{\partial \rho_{K K}}{\partial t}+\frac{\rho_{K K}-\rho_{K K}^{(0)}}{T_{1}}=-i \frac{\mu}{\mathrm{h}} \rho_{K, K-1} \frac{\bar{E}^{*}}{2}+\frac{J_{K}(V, T)}{e}+\text { c. c., } \\
& \frac{\partial \rho_{11}}{\partial t}+\frac{\rho_{11}-\rho_{11}^{(0)}}{T_{1}}=i \frac{\mu}{\mathrm{h}} \rho_{21} \frac{\bar{E}^{*}}{2}+\frac{J_{1}(V, T)}{e}+\text { c.c., }
\end{aligned}
$$

Where $V(t)=e L E(t), J_{k}$ is injection current in a nanotube, which consists of electrons with energy $\bar{\varepsilon}$ in the multicascade mechanism of teraherz radiation generation in the laser; or current $J_{k}$ appears because of the electron's transition from initial state $\left|k_{\alpha}\right\rangle$ to final state $\left|k_{\beta}\right\rangle$ (see $3 b)$ associated with stimulated absorption). The presence of current in the nanotubes results in the presence of an additional term in the second equation of the system (2.5). If we take into account Eq. (2.5), we can see

$$
\begin{aligned}
& \frac{\partial \rho_{k k}}{\partial t}+\frac{\rho_{k k}-\rho_{k k}^{(0)}}{T_{1}}=\frac{\pi|\mu|^{2} g(\omega)}{2 \mathrm{~h}^{2}}|\bar{E}|^{2} \\
& \times\left(\rho_{k+1, k+1}-2 \rho_{k k}+\rho_{k-1, k-1}\right)+\frac{J_{k}}{e} \\
& g(\omega)=\frac{1 / T_{2}}{\pi\left[1 / T_{2}^{2}+(\Omega-\omega)^{2}\right]},
\end{aligned}
$$

Where $g(\omega)$ is the Lorentzian line shape function [16,41]; index $k_{\alpha}$ points out that the electron energy is equal to $\bar{\varepsilon}=\varepsilon_{k_{\alpha}}$ in condition $\left|k_{\alpha}\right\rangle$. In the case of a multistage radiation generation mechanism when the current is injected into a cascade laser nanotube, equality $\bar{\varepsilon}=\varepsilon_{k_{\alpha}}$ is the energy of electrons injected into the nanoparticles. When the mechanism for obtaining current is 
realized through the transition from initial state $\left|k_{\alpha}\right\rangle$ to final state $\left|k_{\beta}\right\rangle$ associated with stimulated absorption, the current will have the dependence

$$
J_{k}=\sum_{k_{\beta}} \delta_{k, k_{\beta}} J_{k_{\beta}}-\sum_{k_{\alpha}} \delta_{k, k_{\alpha}} J_{k_{\alpha}}
$$

Such a conformity takes into account the changes in quantites $\partial \rho_{k_{\alpha} k_{\alpha}} / \partial t$ and $\partial \rho_{k_{\beta} k_{\beta}} / \partial t$, which are caused because of the transition rate between these states. The first summand means the electrons' injection to finale state $\left|k_{\beta}\right\rangle$, the second one means the reduction of the electrons' transition rate at initial state $\left|k_{\alpha}\right\rangle$. In the case of the multicascade mechanism of radiation generation current will have a dependence

$$
J_{k}=\sum_{k_{\beta}} \delta_{k, k_{\beta}} J_{k_{\beta}} .
$$

\section{2b. Narrow nanoribbons}

We generalise the results obtained in $2 \mathrm{a}$ for the array of the narrow armchair-edge nanoribbons [35]. In [35] basing on the four-point unit cell model (four zigzag chains A, B, C and D; see Fig. 4) for the armchair-edge nanoribbons, we consider the staggered configuration. This model almost coincides with the model used in [31, 42]. In [40] as distinct from [39] the array of chiral nanotubes is viewed. In [35] A, B chains correspond to $\mathrm{gA}$ and $\mathrm{gB}$ chains from [43] when $\mathrm{g}$ is odd; if $\mathrm{g}$ is even, $\mathrm{C}$ and $\mathrm{D}$ from our paper chains correspond to $\mathrm{gA}$ and $\mathrm{gB}$. In [35] the obtained wave functions, equidistant energy levels and dipole matrix element values in the presence of a longitudinal electric field for the armchair-edge nanoribbons were absolutely the same as analogous functions from [36, 39] for the zigzag nanotubes. Thus, later on we consider equations (2.5) and (2.6) to be valid also for the armchair-edge nanoribbons with the staggered configuration.

3. Generation of terahertz radiation in the elongated noninteracting nanotube arrays

3a. Approximation of differential-difference equation for the diagonal elements of the density matrix with a parabolic equation 
We consider the terahertz radiation generation process in the medium based on the array of the noninteracting zigzag nanotubes and armchair-edge nanoribbons). We are going to get energy distribution on the levels of CNTs and nanoribbons arrays. So, we write differential-difference equation (2.6) (see [36]) in the differential form [31, 32, 35]

$$
\frac{\partial \rho(\bar{\varepsilon})}{\partial t}+\frac{\rho-\rho_{0}}{T_{1}}=\frac{\pi \Omega^{2}}{2}|\mu|^{2}|\bar{E}|^{2} g(\omega) \frac{\partial^{2} \rho}{\partial \bar{\varepsilon}^{2}}+\frac{J(V, T, \bar{\varepsilon})}{e}
$$

Where $t$ is time, current function $J(V, T, \bar{\varepsilon})$ in the nanotube or nanoribbon on the right of Eq. (3.1) consists of electrons with energy $\bar{\varepsilon}$. In the further analysis, for convenience, we pass from discrete values to continuous function values $\rho(\bar{\varepsilon})$ and $J(V, T, \bar{\varepsilon})$

$$
\begin{aligned}
& \rho(\bar{\varepsilon})=\sum_{k} \rho_{k k} \delta\left(\bar{\varepsilon}-\bar{\varepsilon}_{k}\right), \quad J(V, T, \bar{\varepsilon})=\sum_{k} J_{k} \delta\left(\bar{\varepsilon}-\bar{\varepsilon}_{k}\right), \\
& \rho_{0}(\bar{\varepsilon})=\sum_{k} \rho_{k k}^{(0)} \delta\left(\bar{\varepsilon}-\bar{\varepsilon}_{k}\right) .
\end{aligned}
$$

Here $\delta\left(\bar{\varepsilon}-\bar{\varepsilon}_{k}\right)$ is the Dirac delta functions, the domain of energy $0<\bar{\varepsilon}<\bar{\varepsilon}_{R}$. Lets consider $L=10^{-7} \mathrm{~m}$ to be the length of nanoparticles. Eq. (3.1) is sufficient for basic condition $\rho(t=0, \bar{\varepsilon})=\rho_{0}(\bar{\varepsilon})$. Taking into account, that on the left border of the energy space there is the basic state (electrons from other states pass to this state with the photons emission), Eq. (3.1 2.1) is sufficient the first boundary condition $\rho(t, \bar{\varepsilon}=0)=\rho_{0}(\bar{\varepsilon}=0)$. On its right border the equation in (3.1) is sufficient for the second boundary condition $\partial \rho /\left.\partial \bar{\varepsilon}\right|_{\bar{\varepsilon}=\bar{\varepsilon}_{R}}=0$. Lorentzian line shape function $g(\omega)$ is determined in the congruence (2.6).

$$
\Omega=\frac{\bar{\varepsilon}_{k}-\bar{\varepsilon}_{k-1}}{\mathrm{~h}}=\frac{\sqrt{3} \pi \gamma_{0} a}{2 \mathrm{~h} L}=\frac{\pi v_{\mathrm{F}}}{L},
$$

where $a=\sqrt{3} b$. The fact that Eq. (3.1 2.1) satisfies the second boundary condition can be explained by the fact that the spectrum becomes inequidistant far from Fermi energy, i.e. the frequency of the transition $\Omega$ between the neighbouring levels will depend on the number of the condition (from quantity $\bar{\varepsilon}$ ). That leads to the fact, that the "diffusion coefficient" significantly reduces so, that value $\rho$ flow reduces on the right border (on the right of Eq. (3.1) we should take into account the dependence of the diffusion coefficient on energy $\bar{\varepsilon}$ ). This means, that value $\partial \rho / \partial \bar{\varepsilon}$ on the right boundary of the domain tends to zero. Therefore we consider great, but finite number of levels and on the right border we will formulate the second boundary condition for Eq. 
(3.1) (Let us consider that quantity $g$ is independent from $\bar{\varepsilon}$ ). In the derivation of Eq. (3.1) the equality is taken into account

$$
\sum_{k} \rho_{k \pm 1, k \pm 1} \delta\left(\bar{\varepsilon}-\bar{\varepsilon}_{k}\right)=\sum_{k} \rho_{k \pm 1, k \pm 1} \delta\left(\bar{\varepsilon} \pm \mathrm{h} \Omega-\bar{\varepsilon}_{k \pm 1}\right)=\rho(\bar{\varepsilon} \pm \mathrm{h} \Omega)
$$

One can obtain the expression for current $J(V, T, \bar{\varepsilon})$ in Eq. (3.1), if the left and right parts in Eq. (2.6) are timed the Dirac Delta function $\delta\left(\bar{\varepsilon}-\bar{\varepsilon}_{k}\right)$, the obtained equation is summed over index k. As the result, we obtain the expression for current from the congruence (3.2). In this work, the Dirac Delta function is approximated by a Gaussian function or a function with a rectangular profile in energy interval $\bar{\varepsilon}_{k}-\mathrm{h} \Omega \leq \bar{\varepsilon} \leq \bar{\varepsilon}_{k}+\mathrm{h} \Omega$

$$
\begin{aligned}
& \delta\left(\bar{\varepsilon}-\bar{\varepsilon}_{k}\right)=\frac{1}{\sqrt{\pi} \Delta \bar{\varepsilon}_{k}} \exp \left[-\left(\frac{\bar{\varepsilon}-\bar{\varepsilon}_{k}}{\Delta \bar{\varepsilon}_{k}}\right)^{2}\right], \\
& \delta\left(\bar{\varepsilon}-\bar{\varepsilon}_{k}\right)=\left\{\begin{array}{c}
1 /(\mathrm{h} \Omega), \quad \bar{\varepsilon} \in\left[\bar{\varepsilon}_{k}-\mathrm{h} \Omega / 2, \bar{\varepsilon}_{k}+\mathrm{h} \Omega / 2\right], \\
0, \quad \bar{\varepsilon} \notin\left[\bar{\varepsilon}_{k}-\mathrm{h} \Omega / 2, \bar{\varepsilon}_{k}+\mathrm{h} \Omega / 2\right],
\end{array}\right.
\end{aligned}
$$

Where $\Delta \bar{\varepsilon}_{k}$ and $\bar{\varepsilon}_{\kappa_{0}}$ are constants.

At $t \rightarrow \infty$, taking into account boundary conditions, the solution (3.1) will take the form of a trapeze

$$
\rho(\bar{\varepsilon})=\left\{\begin{array}{c}
4 \bar{\varepsilon} /\left(\mathrm{h} \Omega \bar{\varepsilon}_{k_{o}}\right), \quad 0 \leq \bar{\varepsilon} \leq \bar{\varepsilon}_{k_{o}} \\
4 /(\mathrm{h} \Omega), \quad \bar{\varepsilon}_{k_{o}}<\bar{\varepsilon} \leq \bar{\varepsilon}_{K_{o}}=\bar{\varepsilon}_{R}
\end{array}\right.
$$

where, in the derivation, it was taken into account (3.6) that, firstly, two electrons can be in one state in accordance with the Pauli principle [44] and, secondly, there are two types of charge carriers (electrons and holes), which contribute to the considered effect. In the derivation of the asymptotic Solution of Eq. (3.6) we also account for the max value of a function to be $\max \rho(\bar{\varepsilon})=4 /(\mathrm{h} \Omega)$ in $\bar{\varepsilon}=\bar{\varepsilon}_{k_{o}}$. We integrate (3.1) in the energy space, as a result, we obtain an expression for the flux of quantity in the energy space, taking into account function $\rho(\bar{\varepsilon})$

$$
\Phi=-\frac{\pi \Omega^{2}}{2}|\mu|^{2}|\bar{E}|^{2} g(\omega) \frac{\partial \rho}{\partial \bar{\varepsilon}},\left.\quad \Phi\right|_{0} ^{\bar{\varepsilon}_{k_{o}}+0}=\frac{2 \pi \Omega^{2}|\mu|^{2}|\bar{E}|^{2} g(\omega)}{\mathrm{h} \Omega \bar{\varepsilon}_{k_{o}}}=\frac{J_{k_{o}}}{e}
$$




\section{3b. Points in the array of elongated nanoparticles for the terahertz radiation generation}

Consider two mechanisms for generating current $J(V, T, \bar{\varepsilon})$ on the right side of (3.1). The first mechanism of generating current, considered in [36], is the injection current in the nanotube in a cascade laser, where an array of nanotubes plays the role of an active region (see Fig. 2; the figure is borrowed from [35]). The second mechanism for obtaining current is realized through a transition from initial state $\left|k_{\alpha}\right\rangle$ to final state $\left|k_{\beta}\right\rangle,\left(k_{\beta}-k_{\alpha}\right)>>1$, associated with stimulated absorption. $\mathrm{A} \mathrm{CO}_{2}$ laser can be used to pump the medium. Next, there is a process of sequential transition from state $\left|k_{\beta}\right\rangle$ to initial state $\left|k_{\alpha}\right\rangle$ by analogy with cascade lasers. Transition rate, associated with stimulated absorbtion of photon $W_{\mathbf{r e}}^{(\mathrm{a})}$ from basic state $\left|k_{\alpha}\right\rangle$ into final state $\left|k_{\beta}\right\rangle$ [45], determines current $J_{k}$ in the congruence (2.7), where $J_{k_{\beta}} / e=-J_{k_{\alpha}} / e=W^{(\mathrm{a})}$.

In the first case, in our model, in Eq. (2.6), total current $I(V, T)$ in a nanoparticle is determined in accordance with the Landauer formula $[46,47]$ for the ballistic transport

$$
I(V, T)=\int J(V, T, \bar{\varepsilon}) d \bar{\varepsilon}, \quad J=\frac{e}{\pi \mathrm{h}}\left[f_{L}(\bar{\varepsilon}, T)-f_{R}(\bar{\varepsilon}, T)\right] \tau(\bar{\varepsilon}, V),
$$

Where $f_{L}(\bar{\varepsilon}, T), \quad f_{R}(\bar{\varepsilon}, T)$ is the function of Fermi distribution on a cathode and anode; $\tau(\bar{\varepsilon}, V)$ is the transmission function depending on the energy $\bar{\varepsilon}$ of the charge carriers and $V$. Current $J(V, T, \bar{\varepsilon})$ is alone determined in expresssions (3.2), i.e. total current equals $I(V, T)=\sum_{k} J_{k}$

In the second mechanism for obtaining the current in Eq. (2.6), we use the transition from initial state $\left|k_{\alpha}\right\rangle$ to final state $\left|k_{\beta}\right\rangle$ due to the stimulated absorption of infrared radiation, for example, using the radiation of a $\mathrm{CO}_{2}$ laser. In such a case the transition rate, associated with stimulated emission of photon $W_{\mathbf{\kappa e}}^{(\mathrm{e})}$ and stimulated absorption of photon $W_{\mathbf{\kappa e}}^{(\mathrm{a})}$ with polarization e into solid angle $d o$ per unit time at the dipole stimulated transition from initial state $\left|k_{\alpha}\right\rangle$ to final state $\left|k_{\beta}\right\rangle$ is associated with the total spontaneous transition rates from an initial state to a final subband $[16 ; 45]$ 


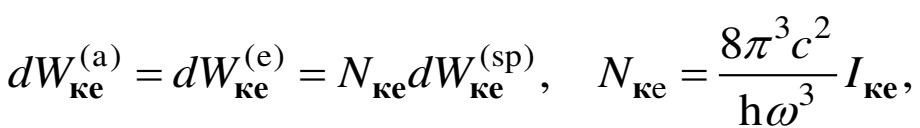

$$
\begin{aligned}
& d W_{\mathbf{k e}}^{(\mathrm{sp})}=W q v=W^{o} \frac{V_{0} \omega^{2} d o}{(2 \pi c)^{3}} d \omega=\frac{\omega^{3}}{2 \pi \mathrm{h} c^{3}}\left|\mathbf{e}^{*} \times \mathbf{d}_{f n}\right|^{2} d o,
\end{aligned}
$$

where $\left|k_{\alpha}\right\rangle=|n\rangle,\left|k_{\beta}\right\rangle=|f\rangle ; d o$ is a solid angle element; $N_{\text {ке }}$ is the number of photons of a given polarization, that fall on one oscillator of the field; the number of oscillators $d v$ in frequency range $d \omega=c d k$ and in solid angle $d o$ is determined by expression $d v=V_{0} k^{2} d k d o /(2 \pi)^{3}$; the sign "×" means the cross product of two vectors; $I_{\text {ке }}$ is spectral intensity in the frequency of electromagnetic radiation with the field polarization vector $\mathbf{e}$ in the direction of wave vector $\mathbf{k} ; \mathbf{d}_{f n}=e z_{f n} \mathbf{e}_{z}$ is the dipole matrix element of the electron in nanopaticles, $\omega=\omega_{f n}=\left(\bar{\varepsilon}_{f}-\bar{\varepsilon}_{n}\right) / \mathrm{h}$; transition rate $W^{\mathscr{\ell}}$ from initial state $|n\rangle$ to final state $|f\rangle$ is obtained from Fermi's golden rule, [48], given by

$$
W^{\circ}=2 \pi\left|V_{f n} / \mathrm{h}\right|^{2} \delta\left(\omega-\omega_{f n}\right)
$$

The computation of the total transition rates from an initial state to a final subband involves the summation over wave vectors from $W^{\mathscr{C}}$ function. Integrating (3.9) over all directions of the solid angle, the transition rate, associated with stimulated absorbtion of photon $W_{\mathbf{K e}}^{(\text {a) }}$ can be written as

$$
W^{(\mathrm{a})}=\int W_{\mathbf{\kappa e}}^{(\mathrm{a})} d o=\int \frac{4 \pi^{2}}{\mathrm{~h}^{2} c} I_{\mathbf{~ к е}}\left|\mathbf{e}^{*} \times \mathbf{d}_{f n}\right|^{2} d o .
$$

when radiation is isotopic and non-polarized ( $I_{\mathbf{K e}}$ doesn't depend on $\mathbf{k e}$ and $\mathbf{e}$ directions), as a result of expression (3.10)intergrating over $d o$ and summation over vector $\mathbf{e}$ we write the transition rate with the use of radiation spectral intensity $I=2 \cdot 4 \pi I_{\text {ке }}$

$$
W^{(\mathrm{a})}=\int W_{\mathbf{\kappa e}}^{(\mathrm{a})} d o=\frac{4 \pi^{2}}{3 \mathrm{~h}^{2} c} I\left|\mathbf{d}_{f n}\right|^{2} .
$$

Transition rate $W_{\mathbf{k e}}^{(\text {a) }}$ from initial state $|n\rangle$ to final state $|f\rangle$ helps us calculate the value of current $J_{k} / e=W^{(a)}$ in Eq. (2.6). Later on for calculating total spectral intensity of the 
radiation we will use the results of [49], in which the pulse length of a $\mathrm{CO}_{2}$ laser and the religion rate of these impulses equal $\tau \approx 1 \mu \mathrm{S}$ and $v \approx 10 \mathrm{kHz}$, desnsity of radiation $N \approx 10 \mathrm{~J} / \mathrm{cm}^{2}$.

\section{Effective Dielectric Function for the Crystal Structure of Nanoparticles}

To investigate the $\mathrm{THz}$ radiation in 3D two-level resonance structure based on crystal lattices of scattering nanoparticles, one should know the effective dielectric function (EDF) $\varepsilon_{\text {eff }}$ of such medium. To define the effective permittivity of a medium, consisting of dielectric particles with dielectric constant $\varepsilon_{1}$, which is also characterized by the depolarization factor $n_{\chi}$, it is necessary to know the dipole moment of the particles (for an ellipsoid see Ref. [50]

$$
p_{\chi}=\alpha_{\chi} E_{\chi}, \quad \mathscr{g}_{\chi}=\frac{\alpha_{\chi}(\omega)}{V \varepsilon_{0}}(\mathrm{SI})=\frac{4 \pi \alpha_{\chi}(\omega)}{V}(\mathrm{CGS})=\frac{\varepsilon_{1}(\omega)-\varepsilon_{2}}{\varepsilon_{2}+n_{\chi}\left(\varepsilon_{1}(\omega)-\varepsilon_{2}\right)}
$$

Where $\alpha_{\chi}$ is the individual particle polarizability, $\varepsilon_{0}$ is a dielectric constant, $\chi=(\mathrm{s}, \mathrm{p})$ means that the polarization is parallel (s-polarization) or perpendicular (p-polarization) to the nanotube's axis.

Enter in Eq. (4.1) by analogy with [51] equivalent isotropic dielectric function $\varepsilon_{1}(\omega)$ for anistropic nanotube, $\varepsilon_{2}$ is the dielectric permittivity of external medium. Suppose that the radius of solid cylinder with isotropic function $\varepsilon_{1}(\omega)$ coincides with the outer radius of nanotube. If this isotropic solid cylinder and the anisotropic nanotube have the same polarizability under the same applied voltage, we call $\varepsilon_{1}(\omega)$ the anisotropic nanotube's equivalent.

The effective medium-approximation (EMA) is valid for densely and randomly distributed particle composites [51]. Maxwell-Garnett's Teoria (MGT) [52, 53] is valid for non-densely and randomly distributed composites of particles. In the geometric model of arrays the effective dielectric functions is defined by the individual particle polarizability, taking into account the geometry of the array (the geometrical factor). The morphologies of both the Maxwell-Garnett theory (MGT) and the array model are not consistent with the dense and random character of this nanotube system. The Maxwell-Garnett approximation is used to analyze the propagation of electromagnetic waves in heterogeneous media to determine the effective dielectric constant of different composite structures, for example, in periodic dielectric systems) [54, 55]). Comparing with the MGT and the array model, the EMA can describe better the experimental data. The 
effective medium approximation (EMA), also called the Bruggeman's theory $[56,57]$, is another method to describe the effective dielectric properties of composites in which the particles of all components are randomly mixed together. This theory has been widely used to explain the dielectric and optical properties of composite materials $[58,59]$ and proved to be valid at all concentrations. In the case of the array model this model is used for an ensemble of separated quantum dots, which are stacked vertically with the finite period [60].

Since volume fraction $f$ is very small, one, basing on the mean field theories, can write effective dielectric function $\varepsilon_{\text {eff }}^{\chi, M G}(\omega)$ with the use of the Maxwell-Garnett formula [51, 61, 62]

$$
\varepsilon_{\mathrm{eff}}^{\chi, \mathrm{MG}}=\varepsilon_{2}\left[1+\frac{f\left(\varepsilon_{1}(\omega)-\varepsilon_{2}\right)}{\varepsilon_{2}+n_{\chi}\left(\varepsilon_{1}-\varepsilon_{2}\right)-f n_{\chi}\left(\varepsilon_{1}-\varepsilon_{2}\right)}\right]
$$

where according to the mean field theories average electric field $E_{\mathrm{av}}$ and electric displacement $D_{\text {av }}$ can be written as

$$
\varepsilon_{e f f}^{\chi, M G}=D_{\mathrm{av}} /\left(\varepsilon_{0} E_{\mathrm{av}}\right), E_{\mathrm{av}}=f E_{1}+(1-f) E_{2}, D_{\mathrm{av}}=f \varepsilon_{1} \varepsilon_{0} D_{1}+(1-f) \varepsilon_{1} \varepsilon_{0} D_{2}
$$

The average inner electric field in a particle can be written with the use of the Laplace's equation

$$
E_{1}=\frac{\varepsilon_{2}}{\varepsilon_{2}+n_{\chi}\left(\varepsilon_{1}(\omega)-\varepsilon_{2}\right)} E_{2}
$$

Maxwell-Garnett formula (4.2) mainly used for dilute systems. If volume fraction $f$ is not small, one can get the effective dielectric function on the basis effective-medium approximation (EMA) [51]

$$
f \frac{\varepsilon_{1}-\varepsilon_{e f f}}{\varepsilon_{e f f}+n_{\chi}\left(\varepsilon_{1}-\varepsilon_{e f f}\right)}+(1-f) \frac{\varepsilon_{2}-\varepsilon_{e f f}}{\varepsilon_{e f f}+n_{\chi}\left(\varepsilon_{2}-\varepsilon_{e f f}\right)}=0 .
$$

In terms of electrodynamics, the discrete structure of array mount of dispersive particles is equal to a continuous dielectric material, characterized by the effective dielectric function (magnetic properties of particles are neglected) [37, 63]) (see also the section "Appendix" in the article under consideration)

$$
\hat{\varepsilon}_{\text {eff }}=\varepsilon_{2}\left[1+\frac{4 \pi}{\Omega} \hat{g}\left(1+\frac{1}{\Omega} \hat{\delta} \cdot \hat{g}\right)^{-1}\right]=\varepsilon_{2}\left[1+\frac{f \mathscr{Q}_{\chi}(\omega)}{1-f B_{\chi} \mathscr{Q}_{\chi}}\right],
$$


Where $B_{\chi}$ is a geometrical factor; $\chi=s, p ; B_{s}$ and $B_{p}$ are the geometrical factors, such, that the polarization is aligned along (s-polarization) or perpendicular (p-polarization) to the nanotube's axis). There is $B_{s}=1 / 3$ when the nanotube centers of a tetragonal lattice are located on the same distance. Ref. (4.6) implies that the polarizability of an individual object is modified by the presence of another via its depolarizing contribution of the electric field acting on this object). Effective dielectric function of the array of nanoparticles depends on the polarizability of individual nanoparticle (4.1) $\mathscr{Q} \ell_{\chi}=\alpha_{\chi} /\left(V \varepsilon_{0}\right)=4 \pi \alpha_{\chi} / V$ and geometrical factor $B_{\chi}$, which, in turn, depends on polarization of incident light $\chi[63,64,65,66]$.

The effective dielectric function formula (4.6) is the Maxwell-Garnett model with the Clausius-Mossotti correction (the arrays geometrical model), since it is based on a rigorous solution of Maxwell's equations under the assumption of a low density of inclusions) [63, 64, 65, 66, 67]. Note, that the denominator in the second term of Eq. (4.6) is the Clausius-Mossotti correction responsible for the electromagnetic interaction of the quantum dots).

In order to determine the effective dielectric function using (4.6), one should use the theoretical dependence of the polarizability of an individual nanotube obtained in [68] (CGSsystem)

$$
\mathscr{\partial} g_{\chi}=\frac{4 \pi \alpha_{\chi}}{V}=-\frac{\omega_{p}^{2}}{\left[\omega^{2}-\bar{\omega}_{0}^{2}+i \omega v\right]}
$$

where $V$ is the volume of spheroid; at the determination of individual nanotube's polarization it is supposed that SWCNT is the prolate spheroid made of an orthorhombic material); $\bar{\omega}_{0}=\omega_{p} \sqrt{n_{\chi}}$ ; in correspondence with [68] for the plasma frequency, there is $\omega_{p}=(4 e / a)\left(v_{F} / \pi \mathrm{h}\right)^{1 / 2}$. There is $B_{s}=1 / 3$ when tetragonal SWCNT the nanotube centers are located on the same distance.

\section{4a. Axisymmetric elongated spheroids}

Elongated nanotubes can be approximated by the axisymmetric elongated spheroids, while greater semiaxis equals to the half of height nanotube $c=L / 2$, transversal sizes of spheroids $a=b$ is taken due to the equality condition of the volumes of particle and spheroid. In this case the depolarization factor along the semi-major axis have form [63] (см. также [50] или [37]) 


$$
n_{3}=\frac{a b c}{2} J_{3}=\frac{a^{2}}{c^{2}}\left[-1+\ln \left(\frac{2 c}{a}\right)\right]<<1,
$$

where $J_{3}$ is the Newtonian potential. The polarizability of spheroid is determined in agreement with (4.1).

\section{4b. Elongated spheroids}

Elongated nanoribbons can be approximated by the axisymmetric elongated spheroids, while greater semiaxis equals to the half of height nanotube $c=L / 2$, transversal sizes of spheroids $a$ and $b$ is taken due to the equality condition of the volumes of particle and spheroid). The depolarization factor along the semi-major axis have form

$$
n_{3}=\frac{a b c}{2} J_{3}=\frac{a b}{c^{2}}\left[-1+\ln \left(\frac{4 c}{a+b}\right)\right]<<1 .
$$

The polarizability of spheroid is determined in agreement with (4.1).

\section{Calculation results}

Based on Ref. (3.1) for the diagonal elements of the density matrix, which can be written in the differential for, we numerically get a solution for function $\rho(n, \bar{\varepsilon})$. Suppose in the numerical solution of Eq. (3.1) the energy definition area as $0 \leq \bar{\varepsilon} \leq \bar{\varepsilon}_{R}$, where $\bar{\varepsilon}_{R}=\alpha_{R} \bar{\varepsilon}_{\kappa_{0}}$, $\bar{\varepsilon}_{\kappa_{0}}=6 \mathrm{~h} \Omega=0.108 \mathrm{eV}$; constant $\alpha_{R}$ has two values $\alpha_{R}=2$ and $\alpha_{R}=4$. On the left border of the energy space for the quantity $\rho(\bar{\varepsilon})$ in (3.1) there is the first boundary condition $\rho(\bar{\varepsilon}=0)$ . On the right border of the energy space for the quantity $\rho(\bar{\varepsilon})$ there is the second boundary condition $\partial \rho /\left.\partial \bar{\varepsilon}\right|_{\bar{\varepsilon}=\bar{\varepsilon}_{R}}=0$. Suppose the hopping integral to be equal to $\gamma_{0}=2.7 \mathrm{eV}$; $L=10^{-7} \mathrm{~m}, \quad|\mu|=3.24 \times 10^{-27} \mathrm{Q} \times \mathrm{m}, \quad|\bar{E}|=2.2 \times 10^{5} \mathrm{~V} / \mathrm{m}, \quad T_{1}=T_{2}=3 \times 10^{-12} \mathrm{~s}$, $k T=0.0258 \mathrm{eV}(T=300 \mathrm{~K})$. The transition frequency between the adjacent levels is $\Omega=2.7 \times 10^{13} \mathrm{~s}^{-1}$. The Lorentzian line shape function $g(\omega)$ is defined in Eq. (2.6). In Eq. (3.11) and (3.2) function $\rho_{0}(\bar{\varepsilon})$ and the eigenvalues of energy states of charge $\bar{\varepsilon}_{k}$ carriers are in form of 


$$
\begin{aligned}
& \rho_{0}(\bar{\varepsilon})=\sum_{k} \rho_{k k}^{(0)} \delta\left(\bar{\varepsilon}-\bar{\varepsilon}_{k}\right), \quad \rho_{k k}^{(0)}=\frac{1}{1+\exp \left[\bar{\varepsilon}_{k} /(k T)\right]} \\
& \bar{\varepsilon}_{k}=\frac{3 \pi b\left|\gamma_{0}\right|}{4 L}(1+2 k)=\frac{\mathrm{h} v_{\mathrm{F}}}{4 L}(1+2 k)=\mathrm{h} \Omega,
\end{aligned}
$$

where $U_{\mathrm{F}}$ is Fermi velocity. Write the initial condition of the differential equation (3.1) is in form of $\rho(t=0, \bar{\varepsilon})=\rho_{0}(\bar{\varepsilon})$.

In the case of the multicascade mechanism we suppose the magnitude in nanotubes in Eq. (2.8) to be equal to $J_{k_{0}}=8 \times 10^{-6} \mathrm{~A}$. The magnitude in nanotubes, which can be used as a nonlinear amplification region of radiation in cascade lasers, was borrowed from Ref. [36] [36]. In Ref. [36] current for the ballistic transport is determined in accordance with the Landauer formula (3.8) $[46,47]$.

In Fig. 1 and 2 there is the dependence of function $\not(t), \bar{\varepsilon})$ on electron energy $\bar{\varepsilon}$ and time $t$ in $\alpha_{R}=2$ and $\alpha_{R}=4$, where $\beta(t t, \bar{\varepsilon})=\rho(t, \bar{\varepsilon})-\rho_{0}(\bar{\varepsilon})$, where function $\beta(t t, \bar{\varepsilon})$ is valid for the equation with the the initial condition and boundary conditions

$$
\begin{aligned}
& \frac{\partial \beta(\bar{\varepsilon})}{\partial t}+\frac{\beta 0}{T_{1}}=\frac{\pi \Omega^{2}}{2}|\mu|^{2}|\bar{E}|^{2} g(\omega) \frac{\partial^{2} \not b o}{\partial \bar{\varepsilon}^{2}}+\frac{J(V, T, \bar{\varepsilon})}{e} \\
& +\frac{\pi \Omega^{2}}{2}|\mu|^{2}|\bar{E}|^{2} g(\omega) \frac{1}{4 \mathrm{~h} \Omega k^{2} T^{2}} \frac{\operatorname{sh}[\bar{\varepsilon} /(2 k T)]}{c^{3}[\bar{\varepsilon} /(2 k T)]}, \\
& \beta(b t=0, \bar{\varepsilon})=0, \quad \beta p_{\bar{\varepsilon}=0}=0,\left.\quad \frac{\partial \beta \phi}{\partial \bar{\varepsilon}}\right|_{\bar{\varepsilon}=\alpha_{R} \bar{\varepsilon}_{\kappa_{0}}}=-\frac{1}{4 \mathrm{~h} \Omega k T} \operatorname{ch}^{-2}\left(\frac{\alpha_{R} \bar{\varepsilon}_{\kappa_{0}}}{2 k T}\right),
\end{aligned}
$$

where delta function $\delta\left(\bar{\varepsilon}-\bar{\varepsilon}_{k}\right)$ in (5.2) and (5.1) are approximated by the Gauss function from (3.5); magnitude $\Delta \bar{\varepsilon}_{k}$ in the Gauss function is equal to $\Delta \bar{\varepsilon}_{k}=\mathrm{h} \Omega / 5$. 


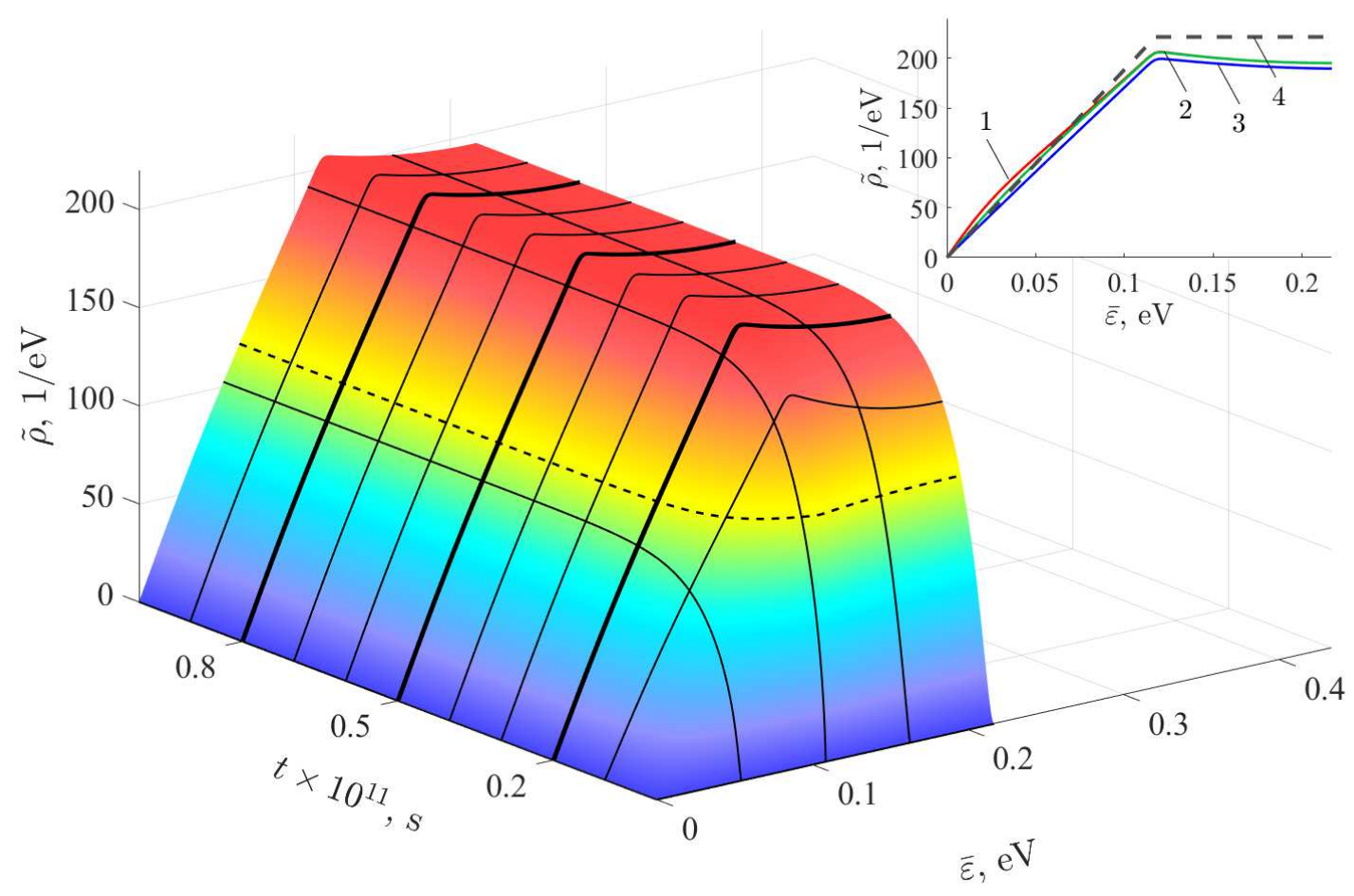

Fig. 1. Dependence of functions $\beta \ll$ on electron energy $\bar{\varepsilon}$ in time $t$ at $\alpha_{R}=2$. Curves $1,2,3$ in the inset correspond to a solution at $T=150,300,600 \mathrm{~K}$ at time moment $t=10^{-11} \mathrm{~s}$ respectively; curve 4 corresponds to the analytical solution (3.6). The dashed-line represents points, in which the values of variable $\beta_{l}$ are equal to constant $\beta_{c}=120 /(\mathrm{eV})$.

The inset of Fig. 1 shows dependence of function $\not(b t, \bar{\varepsilon})$ from Eq. (5.2) at time moment $t=10^{-11} \mathrm{~s}$ in the steady mode region at different values of temperature $T=150 \mathrm{~K}, T=300 \mathrm{~K}$ and $T=600 K$ (curves 1, 2 and 3). Dashed-line curve 4 corresponds to the analytical solution (3.6)). 


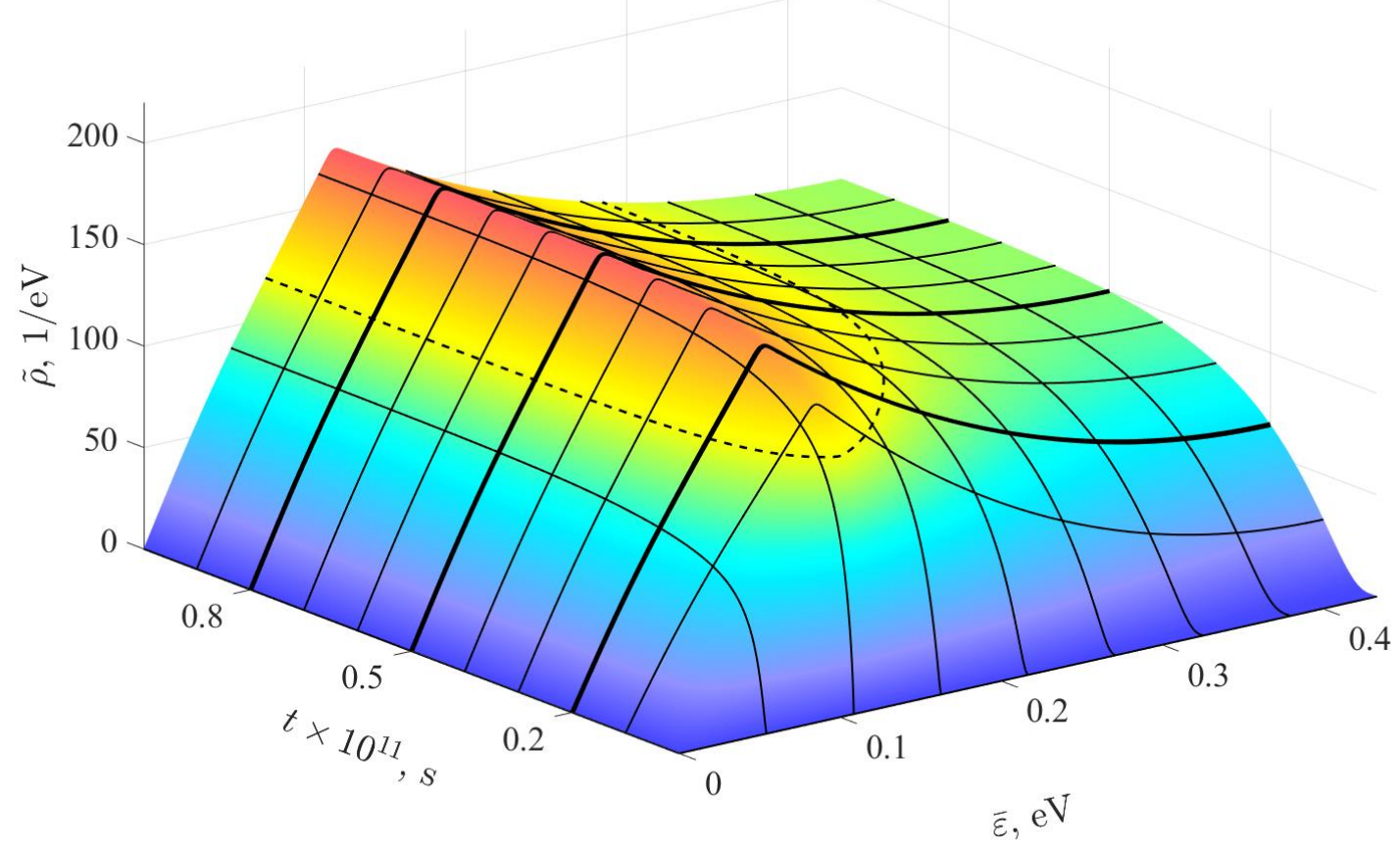

Fig. 2. Dependence of functions $\beta$, on electron energy $\bar{\varepsilon}$ and time $t$ at $\alpha_{R}=4$.

In the mechanism of generation of radiation, when the current is obtained during the transition from the initial state to the final state, associated with stimulated absorption, we will assume that the current determined in (2.7) will have the dependence

$$
J_{k}=\delta_{k, k_{0}} J_{k_{0}}-\delta_{k, k_{1}} J_{k_{1}}
$$

where $k_{0}=6, k_{1}=0$. In (3.11) we wrote transition rate $W^{(\mathrm{a})}$ with the help of radiation spectral intensity $I=2 \cdot 4 \pi I_{\text {ке }}$. The same dependence for radiation spectral intensity is obtained in Ref. [16] for the two-level system, in which dependence of spectral intensity $I$ is expressed with intensity $I_{0}$ of radiation $I \approx I_{0} / \Delta \omega \sim I_{0} T_{2}$.

For calculating the radiation spectral intensity we use the results from Ref. [49], in which the pulse-length of a $\mathrm{CO}_{2}$-laser and the repetition rate of these impulses are equal to $\tau \approx 1 \mu \mathrm{S}$ and $v \approx 10 \mathrm{kHz}$, density of the radiation $N \approx 10 \mathrm{~J} / \mathrm{cm}^{2}$. Thus $I_{0}=N / \tau \approx 10^{11} \mathrm{~J} /\left(\mathrm{m}^{2} \times \mathrm{s}\right)$. Taking into account the expression for the dipole matrix element of laser transition

$$
\left(d_{z}\right)_{k_{1}, k_{0}}=e(z)_{k_{1}, k_{0}}=-2 L /\left[\pi^{2}\left(k_{0}-k_{1}\right)^{2}\right],
$$


presented after the system of Eq. (2.2), from (3.11) we get the values for the magnitude of transition rate $W^{(\mathrm{a})}$,

$$
\begin{aligned}
& W^{(\mathrm{a})}=\frac{4 \pi^{2}}{3 \mathrm{~h}^{2} c} \frac{N T_{2}}{\tau}\left|\mathbf{d}_{f n}\right|^{2} \approx 9.26 \times 10^{13} \mathrm{~s}^{-1}, \\
& W^{(\mathrm{a})}=\frac{J_{k_{0}}}{e}=\frac{J_{k_{1}}}{e}, \quad\left|\mathbf{d}_{f n}\right|^{2}=e^{2}\left|z_{n f}\right|^{2}=\alpha \mathrm{h} c\left|z_{n f}\right|^{2},
\end{aligned}
$$

where $\alpha=1 / 137$ is the constant of a thin structure.

In calculations we consider the magnitude hoping integral to be $\gamma_{0}=2.7 \mathrm{eV}, L=10^{-7} \mathrm{~m}$, as in the case of the multicascade mechanism of radiation generation. In contrast to the case of the multicascade mechanism of radiation generation, the magnitude of current and field intensity in Eq. (2.8) are equal to $J_{k_{0}}=1.48 \times 10^{-5} \mathrm{~A},|\bar{E}|=3.26 \times 10^{5} \mathrm{~V} / \mathrm{m}$.

In Fig. 3 and 4 there is dependence of function $\rho(t, \bar{\varepsilon})$ on electron energy $\bar{\varepsilon}$ and time $t$ at $\alpha_{R}=2$ and $\alpha_{R}=4$. In calculations Eq. (5.2) was being numerically solved, taking into account given boundary conditions and initial condition $\quad \beta(\not t=0, \bar{\varepsilon})=0$, where $\rho(t, \bar{\varepsilon})=\beta(b t, \bar{\varepsilon})+\rho_{0}(\bar{\varepsilon})$. The inset of Fig. 3 shows dependence $\rho(t, \bar{\varepsilon})$ at time moments $t=0.004 \times 10^{-11} \mathrm{~s}$ (curve 2) and $t=10^{-11} \mathrm{~s}$ in the steady mode region (curve 1). Curve 3 corresponds to the analytical solution (3.6 2.6). 


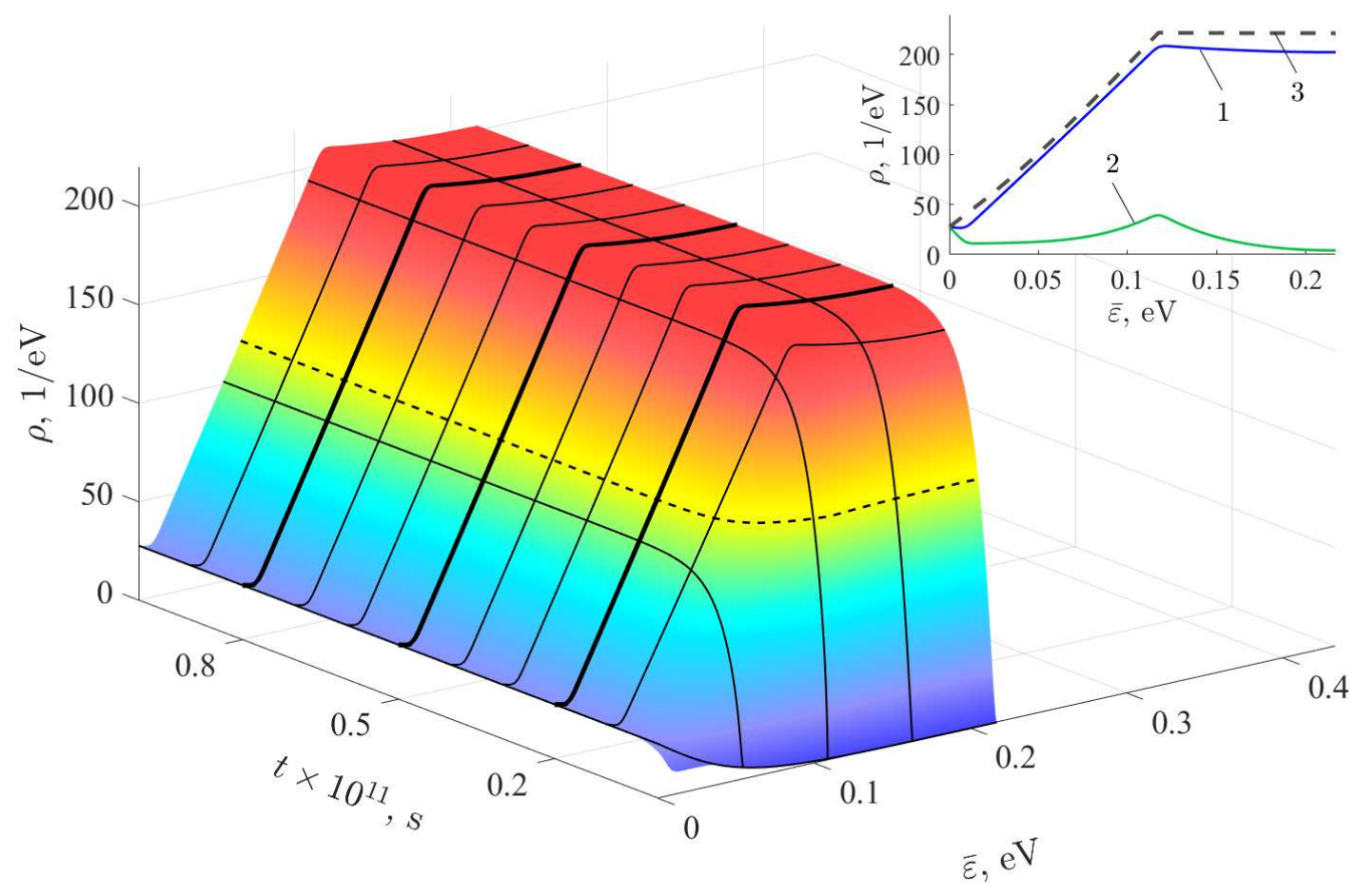

Fig. 3. Dependence of functions $\rho(\bar{\varepsilon})$ on electron energy $\bar{\varepsilon}$ and time $t$ at $\alpha_{R}=2$. The inset shows dependence $\rho$ on $\bar{\varepsilon}$ at time moment $t=10^{-12}$ s (curve 1) and $t=0.004 \times 10^{-11}$ s (curve 2). Curve 3 corresponds to the analytical solution (3.6).

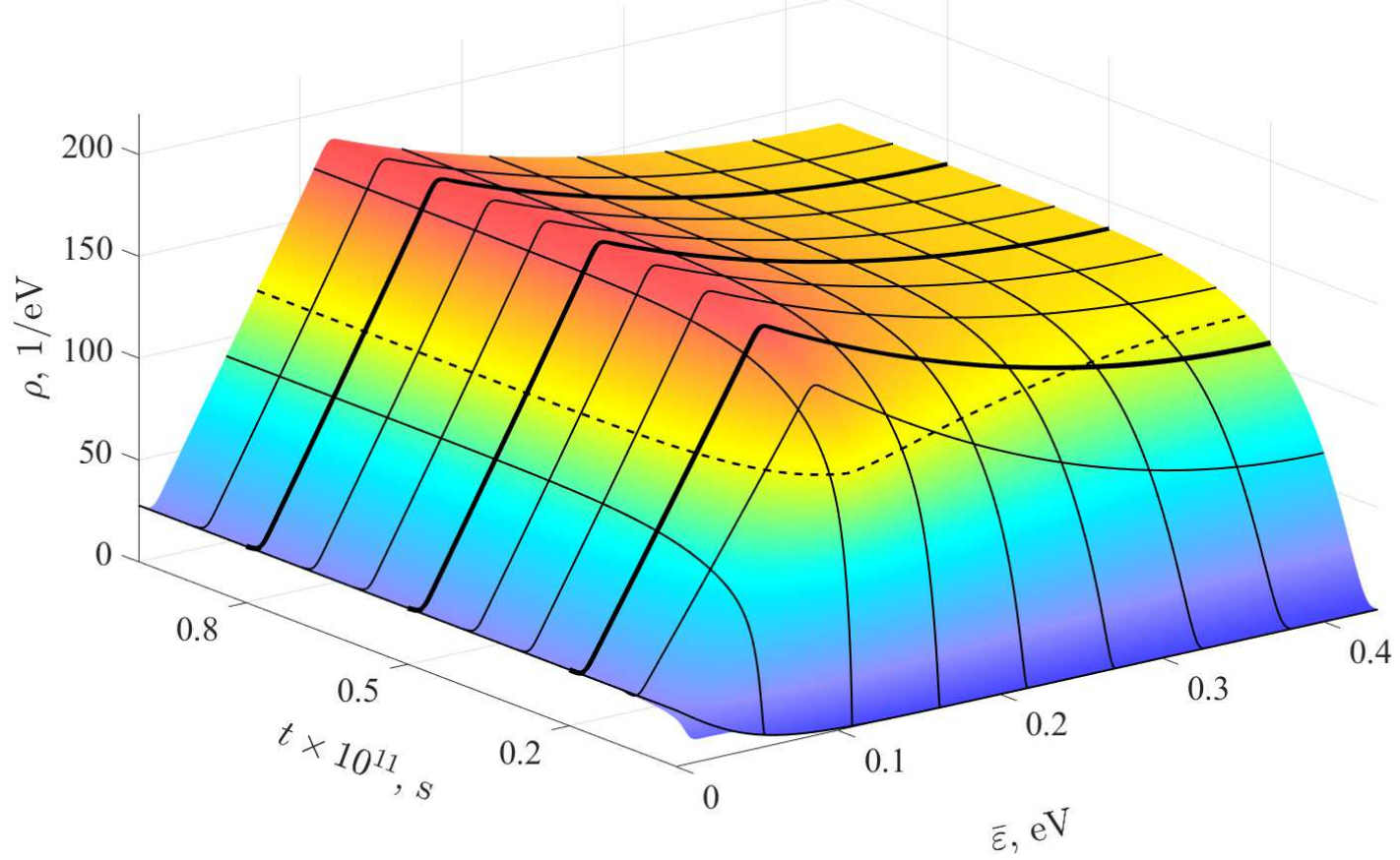

Fig. 4. Dependence of functions $\rho(\bar{\varepsilon})$ on elrectron energy $\bar{\varepsilon}$ and time $t$ at $\alpha_{R}=4$. 
In Fig. 5 and 6 on the basis EMA [51] there is dependence of imaginary $\operatorname{Im} \varepsilon_{\text {eff }}$ and real $\operatorname{Re} \varepsilon_{\text {eff }}$ parts of the effective dielectric function $\varepsilon_{\text {eff }}$ on frequency in intervals $10^{12} \leq \omega / 2 \pi \leq 5 \times 10^{12} \mathrm{~s}^{-1}$ and ${ }^{12} \leq \omega / 2 \pi \leq 10^{13} \mathrm{~s}^{-1}$, respectively, for the bundle of CNT. Dottedline curves $1,2,3$ correspond to the case where volume ratio $f=1.44 \times 10^{-3}$ conforms to a situation, in which an average value between nanotubes is equal to $30 \mathrm{~nm}$; solid-line curves 4,5 , 6 correspond to the case where volume fraction $f=3.9 \times 10^{-5}$ conforms to a situation, in which an average value of the distance between nanotubes is equal to $100 \mathrm{~nm}$; dashed-line curves 7,8 , 9 correspond to the case where volume fraction $f=1.44 \times 10^{-6}$ conforms to a situation, in which an average value of the distance between nanotubes is equal to $300 \mathrm{~nm}$. Curves 1, 4 and 7 (in green) conform to the case of $v=3 \times 10^{11} \mathrm{~s}^{-1}$; curves 2,5 and 8 (in blue ) conform to the case of $v=3 \times 10^{12} \mathrm{~s}^{-1}$; curves 3,6 and 9 (in red) conform to the case of $v=3 \times 10^{13} \mathrm{~s}^{-1}$. Vertical solidline curve 10 in Fig. 5 and 6 conforms to frequency $\omega_{0} / 2 \pi$; vertical dashed-line curves 10 and 11 conform two radiation frequencies of $\mathrm{CO}_{2}$ laser $c / \lambda_{1}$ and $c / \lambda_{2}$, where $\lambda_{1}=10.6 \mu \mathrm{m}$ and $\lambda_{2}=9.4 \mu \mathrm{m}$. Изображенные на рис. 5 на иконке (the inset) кривые представляют собой продолжение кривых 1-9 в интервале частот $10^{12} \leq \omega / 2 \pi \leq 10^{13} \mathrm{~s}^{-1}$ и $10^{12} \leq \omega / 2 \pi \leq 5 \times 10^{12} \mathrm{~s}^{-1}$, соответственно (The curves, depicted in the inset of Fig. 5 , are the continuation of curves $1-9$ in frequency interval $10^{12} \leq \omega / 2 \pi \leq 10^{13} \mathrm{~s}^{-1}$ and $10^{12} \leq \omega / 2 \pi \leq 5 \times 10^{12} \mathrm{~s}^{-1}$, respectively. The vertical curve in the inset corresponds to the radiation wave length $\omega / 2 \pi \Omega, \Omega=2.7 \times 10^{13} \mathrm{~s}^{-1}$. In Fig. 6 curves $1,2,5$, evicted in the inset, are practically the same.

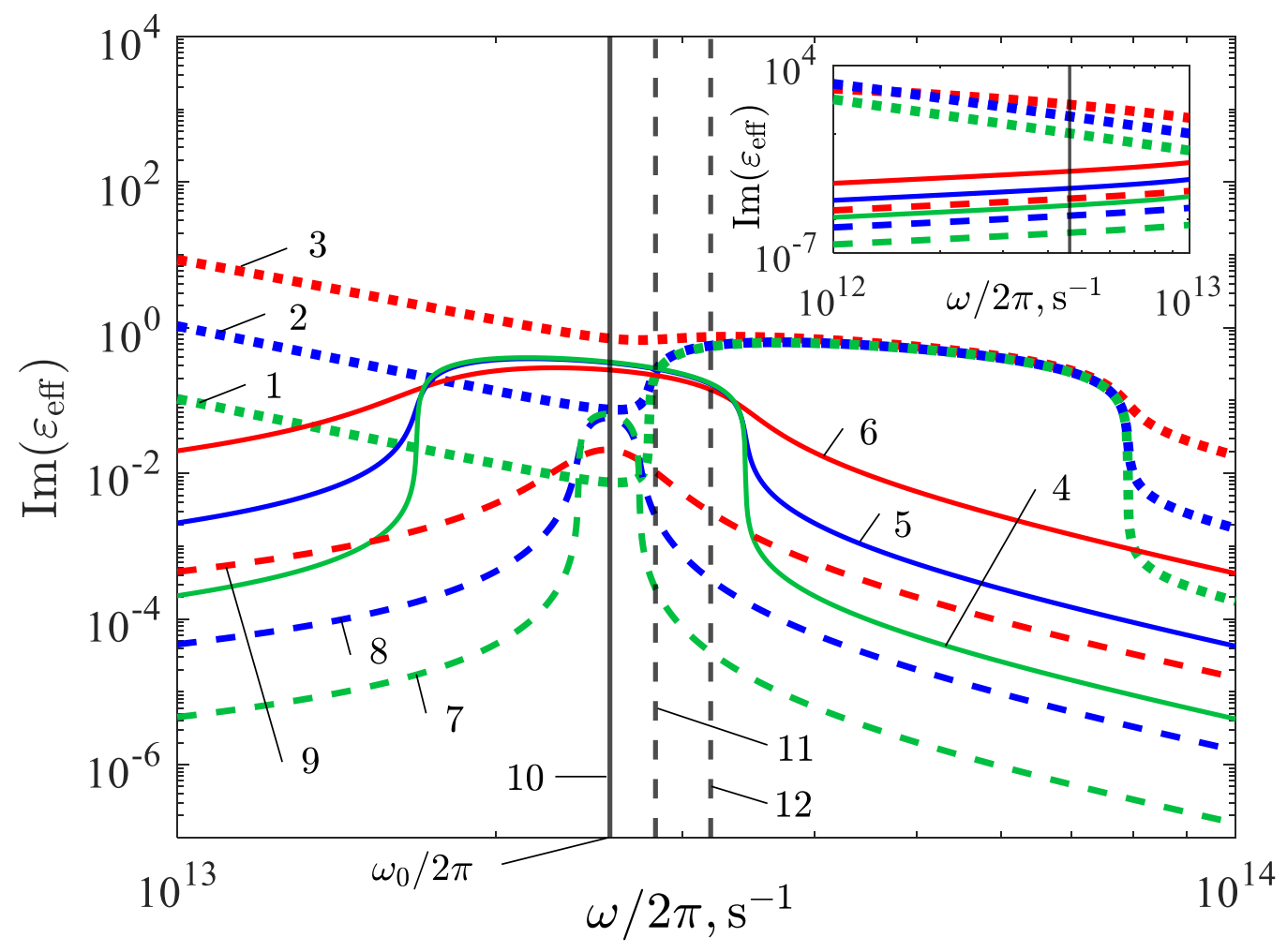


Fig. 5. Dependence of an imaginary part of effective dielectric function $\varepsilon_{\text {eff }}$ on frequency on the basis EMA at volume fraction $f=1.44 \times 10^{-3} ; 3.9 \times 10^{-5} ; 1.44 \times 10^{-6}$ of the CNTs bundle.

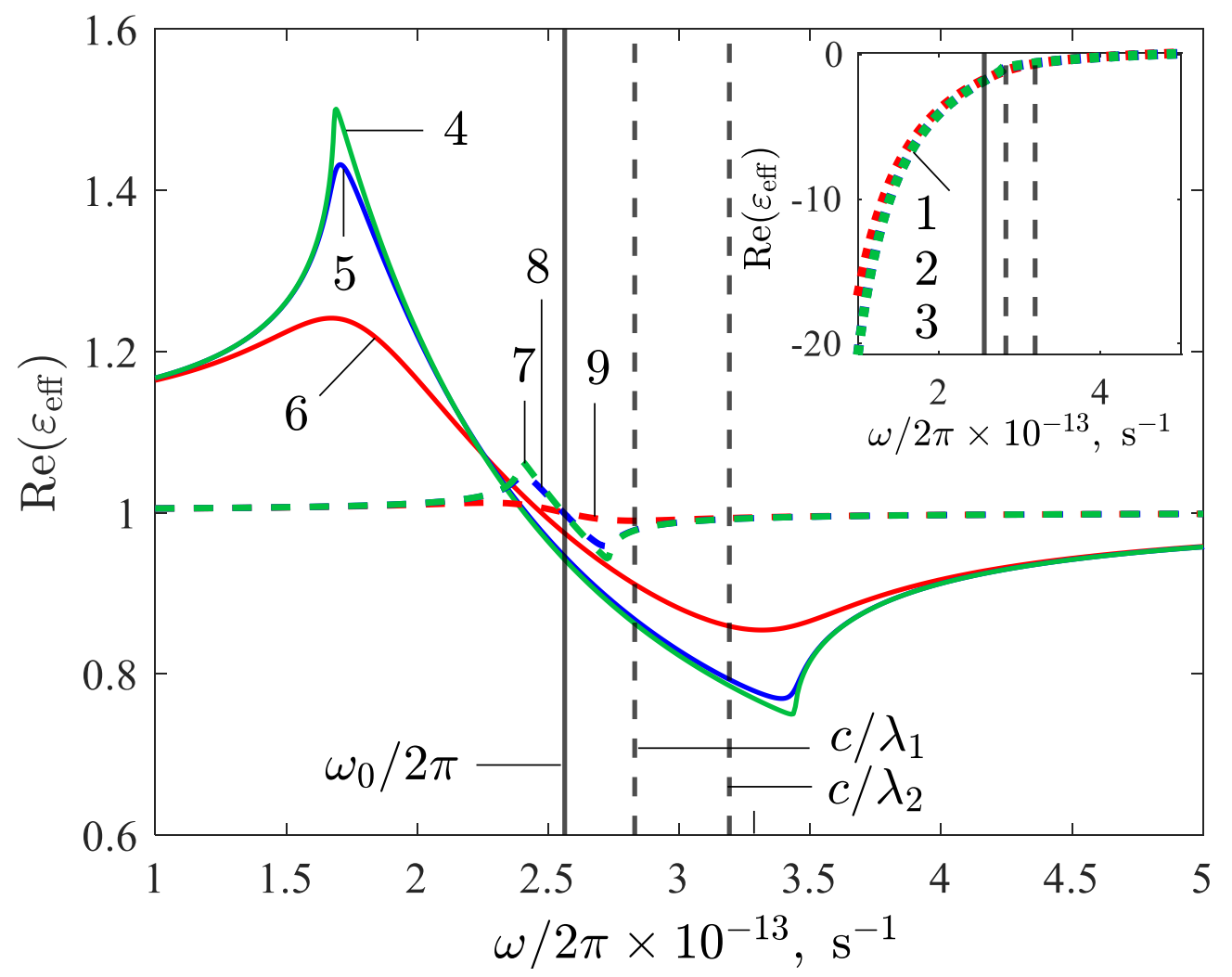

Fig. 6. Dependence of a real part of effective dielectric function $\varepsilon_{\text {eff }}$ on frequency on the basis EMA at volume fraction $f=1.44 \times 10^{-3} ; 3.9 \times 10^{-5} ; 1.44 \times 10^{-6}$ of the CNTs bundle.

In Fig. 7 and 8 there is a numerically obtained dependence of imaginary $\operatorname{Im}\left(\varepsilon_{\text {eff }}\right)$ and real $\operatorname{Re}\left(\varepsilon_{\text {eff }}\right)$ parts of the effective dielectric function on the frequency in intervals $10^{13} \leq \omega / 2 \pi \leq 5 \times 10^{14} \mathrm{~s}^{-1}$ and $2.45 \times 10^{13} \leq \omega / 2 \pi \leq 2.7 \times 10^{13} \mathrm{~s}^{-1}$, respectively, in the array of CNT, basing on the Maxwell-Garnett's Thery (MGT). In Fig. 7 and 8 doted-line curves 1, 2, 3 correspond to the case of $f=1.44 \times 10^{-3}$ (an avaerage value of the distance between nanotubes is $30 \mathrm{~nm}$ ); solid-line curves $4,5,6$ correspond to the case of $f=3.9 \times 10^{-5}$ (an avaerage value of the distance between nanotubes is $100 \mathrm{~nm}$ ); dashed-line curves $7,8,9$ correspond to the case of $f=1.44 \times 10^{-6}$ (an avaerage value of the distance between nanotubes is $300 \mathrm{~nm}$ ). The 
vertical-line curve in the inset corresponds to radiation wave length $\omega / 2 \pi \Omega, \quad \Omega=2.7 \times 10^{13} \mathrm{~s}^{-1}$

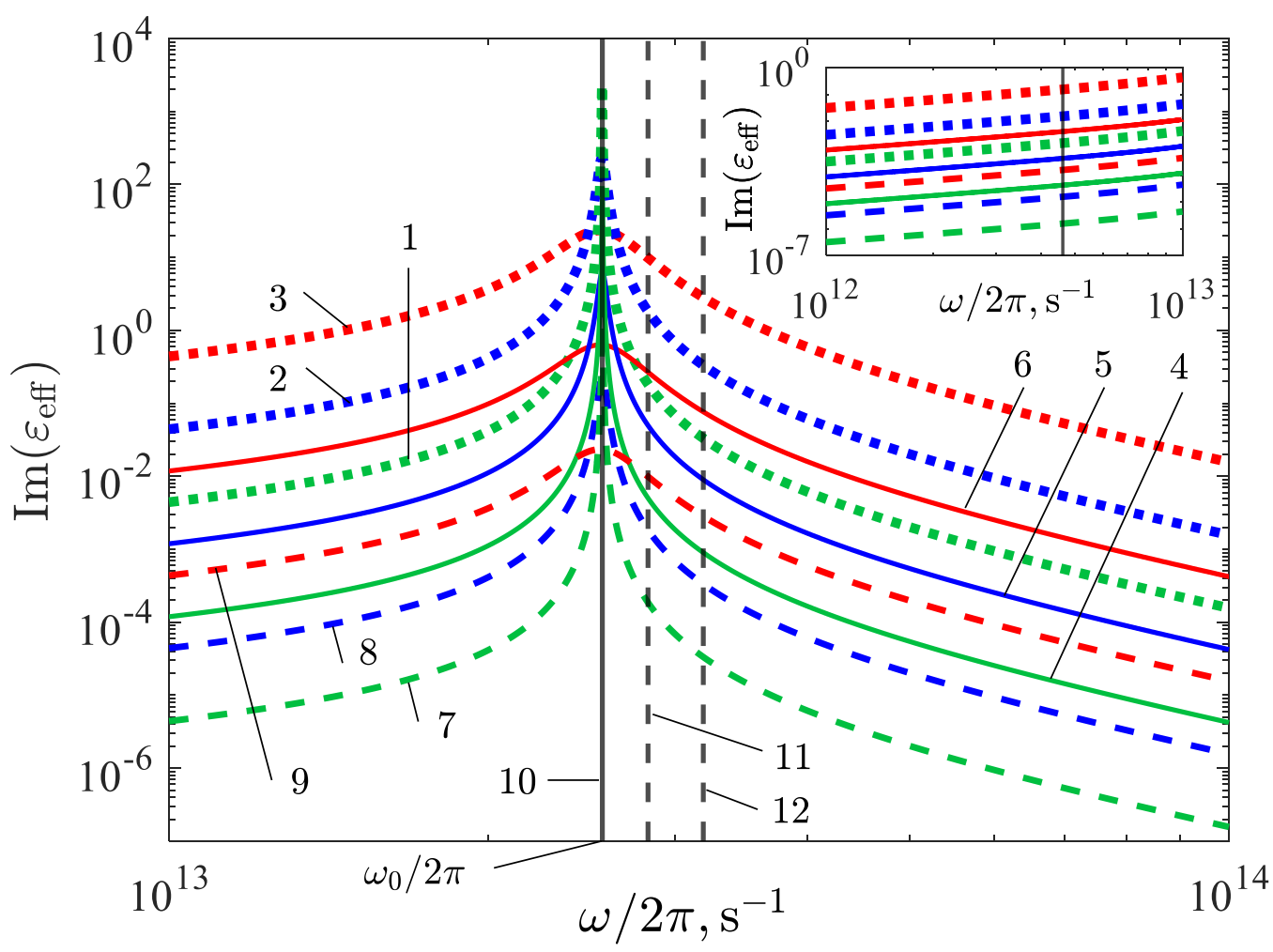

Fig. 7 Dependence of and imaginary part of effective dielectric function $\varepsilon_{\text {eff }}$ on the frequency, based on the Maxwell-Garnett's Theory (MGT), at volume fraction $\left.f=1.44 \times 10^{-3} ; 3.9 \times 10^{-5} ; 1.44 \times 10^{-6}\right)$.

Curves 1, 4 and 7 (in green) in Fig. 7 correspond to the case of $v=3 \times 10^{11} \mathrm{~s}^{-1}$; curves 2, 5 and 8 (in blue) correspond to the case of $v=3 \cdot 10^{12} \mathrm{~s}^{-1}$; curves 3,6 and 9 (in red) correspond to the case of $v=3 \times 10^{13} \mathrm{~s}^{-1}$. Solid-line curve 10 in Fig. 7 and 8 corresponds to frequency $\omega_{0} / 2 \pi \approx 2.56 \times 10^{13} \mathrm{~s}^{-1}$; vertical dashed-line curves 10 and 11 in Fig. 7 correspond to two radiation frequencies of a $\mathrm{CO}_{2}$ laser: $c / \lambda_{1}$ and $c / \lambda_{2}$, where $\lambda_{1}=10.6 \mathrm{~m} \mu$ and $\lambda_{2}=9.4 \mathrm{~m} \mu$. Depicted in the inset of Fig. 7 curves are the continuation of curves 1-9 in the frequency interval. The results of calculations have been obtained with the use of the Maxwell-Garnett formula (4.2). In Fig. 8 the value of function $\operatorname{Re} \varepsilon_{\text {eff }}$ at $\omega / 2 \pi=10^{12}$ correspond to the dotted-, solid- and dashedline curbes and are equal to $5.38,1.12,1.004$. 


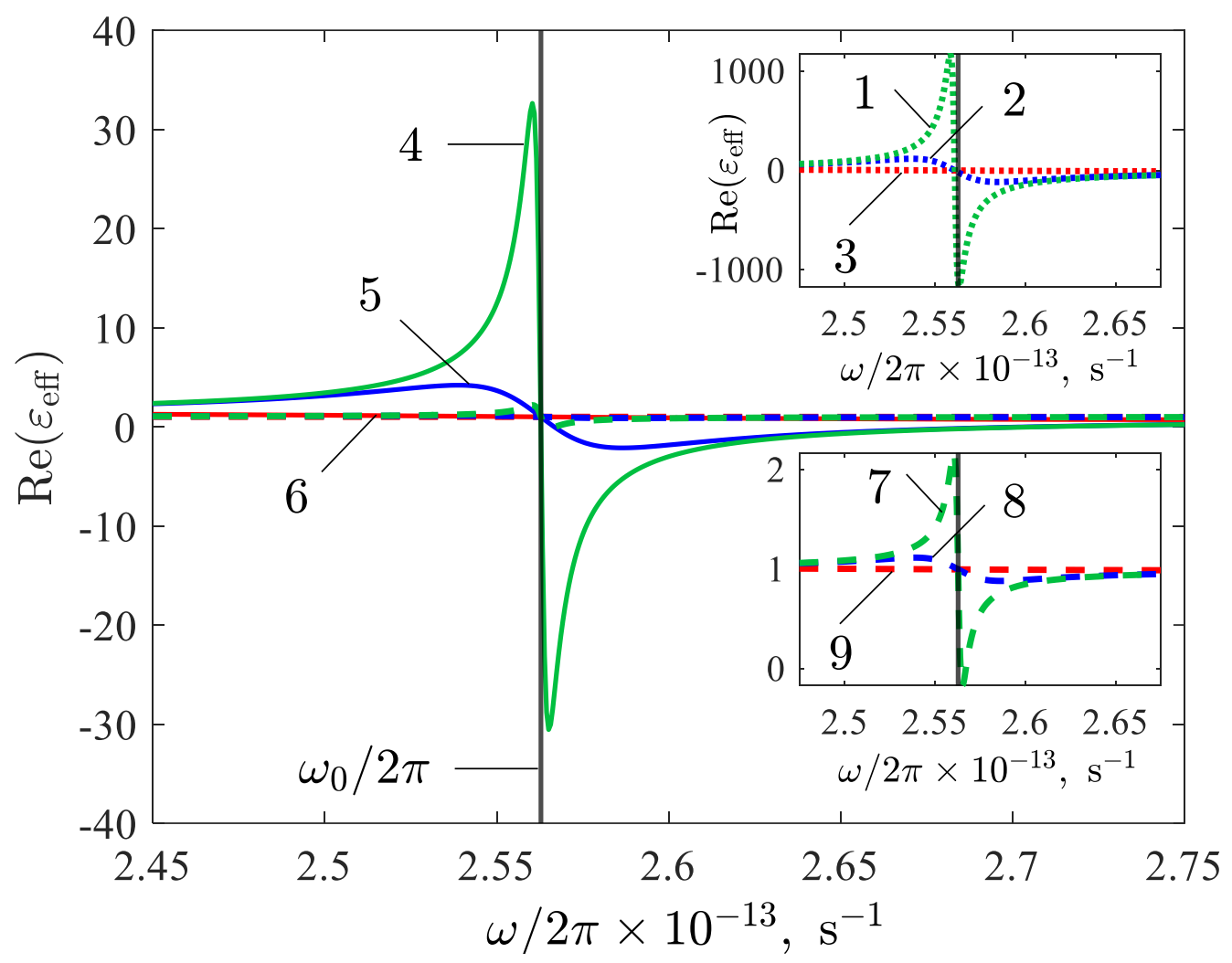

Fig. 8. Dependence of a real part of effective dielectric function $\varepsilon_{\text {eff }}$ on frequency, based on the MGT at volume fraction $f=1.44 \times 10^{-3} ; 3.9 \times 10^{-5} ; 1.44 \times 10^{-6}$.

In Fig. 9 and 10 we can see dependence of imaginary $\operatorname{Im}\left(\varepsilon_{\text {eff }}\right)$ and real $\operatorname{Re} \varepsilon_{\text {eff }}$ parts of effective dielectric function $\varepsilon_{\text {eff }}$ on the frequency in intervals $10^{13} \leq \omega / 2 \pi \leq 10^{14} \mathrm{~s}^{-1}$ and $2.40 \times 10^{13} \leq \omega / 2 \pi \leq 2.75 \times 10^{13} \mathrm{~s}^{-1}$, respectively, based on the Maxwell-Garnett model with the Clausius-Mossotti correction (the arrays geometrical model; see formula (4.6) and section "Appendix") in an array with a tetragonal lattice CNT, which is based on a strict solution of the Maxwell equations in the offered model of the law volume inclusion. In Fg. 9 and 10 dotted-line curves 1, 2, 3 correspond to the case where the distance between nantubes is equal to $\Delta x \times \Delta y \times \Delta z=20 \times 20 \times 67.5 \mathrm{~nm}$ (the volume fraction is $f=1.44 \times 10^{-3}$, the geometrical factor is $\left.B_{S}=0.44\right)$; solid-line curves $4,5,6$ correspond to the case where the distance between nanotubes is equal to $\Delta x \times \Delta y \times \Delta z=71 \times 71 \times 198.4 \mathrm{~nm}$ (the volume fraction is $f=3.9 \times 10^{-5}$, the geometrical factor is $B_{s}=0.53$ ); dashed-line curves $7,8,9$ correspond to the case where the distance between nanotubes is equal to $\Delta x \times \Delta y \times \Delta z=300 \times 300 \times 300 \mathrm{~nm}$ 
(the volume fraction is $f=1.44 \times 10^{-6}$, the geometric factor is $B_{s}=1 / 3$. Curves 1,4 and 5 (in green) in Fig. 9 correspond to the case of $v=3 \times 10^{11}$; curves 2, 5 and 8 (in blue) correspond to the case of $v=3 \cdot 10^{12}$; curves 3,6 and 9 (in red) correspond to the case of $v=3 \cdot 10^{13}$. Solidline curve 10 in Fig. 9 and 10 corresponds to frequency $\omega_{0} / 2 \pi$; vertical dashed-line curves 11 and 12 in Fig. 9 correspond to two radiation frequencies $c / \lambda_{1}$ and $c / \lambda_{2}$ of a $\mathrm{CO}_{2}$ laser, where $\lambda_{1}=10.6 \mathrm{~m} \mu$ and $\lambda_{2}=9.4 \mathrm{~m} \mu$. In Fig. 10 all the curves cross at $\operatorname{Re} \varepsilon=1$. The values of function $\operatorname{Re} \varepsilon_{\text {eff }}$ at $\omega / 2 \pi=10^{12}$, corresponding to the dotted-, solid- and dashed-line curves, are equal to $-3.77 ; 1.13 ; 1.004$.

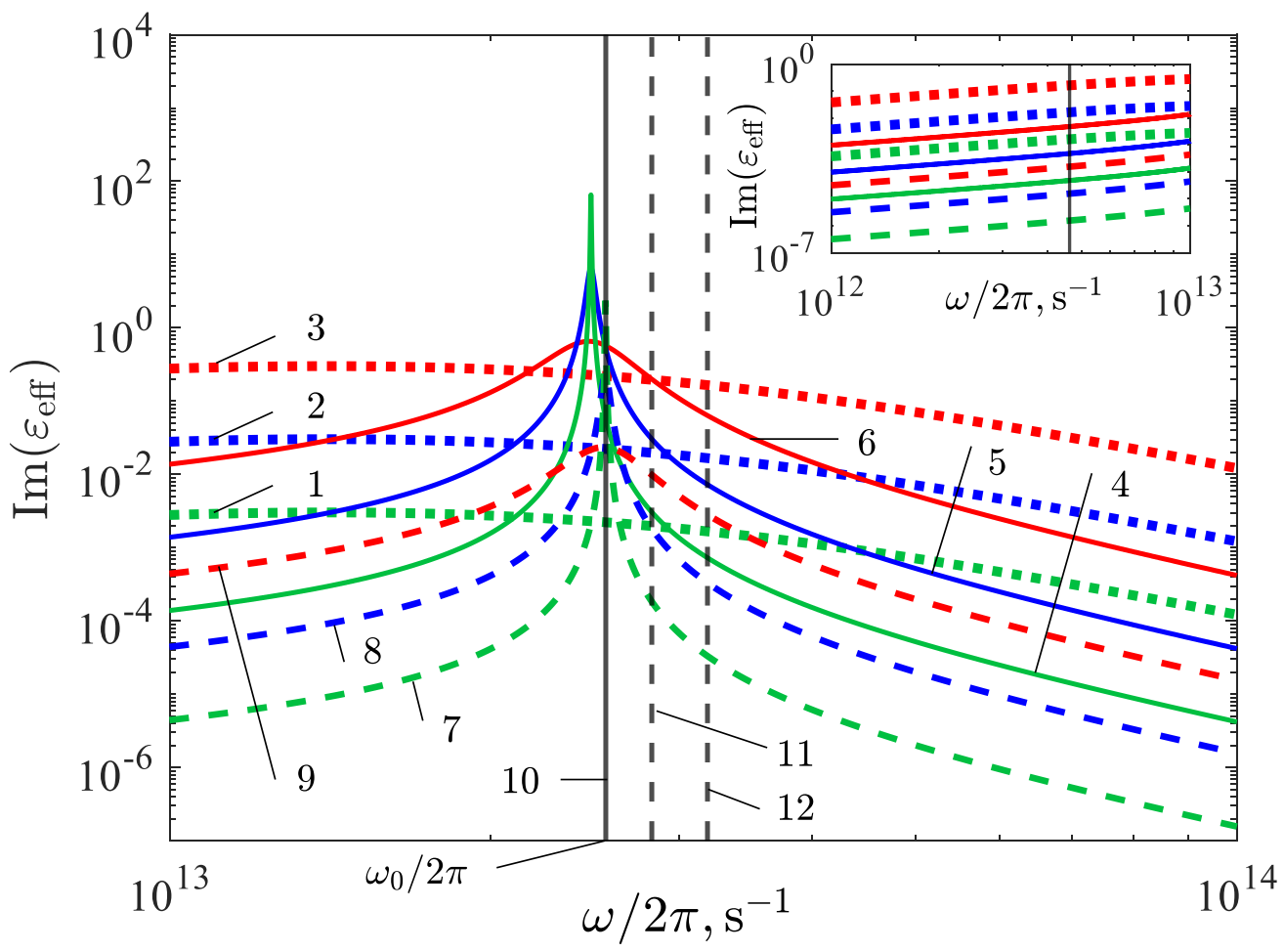

Fig. 9. Dependence of an imaginary part of effective dielectric function $\varepsilon_{\text {eff }}$ on the CNT array frequency, based on the Maxwell-Garnett's model corrected by Clausius-Mossoti (the arrays geometrical model). 


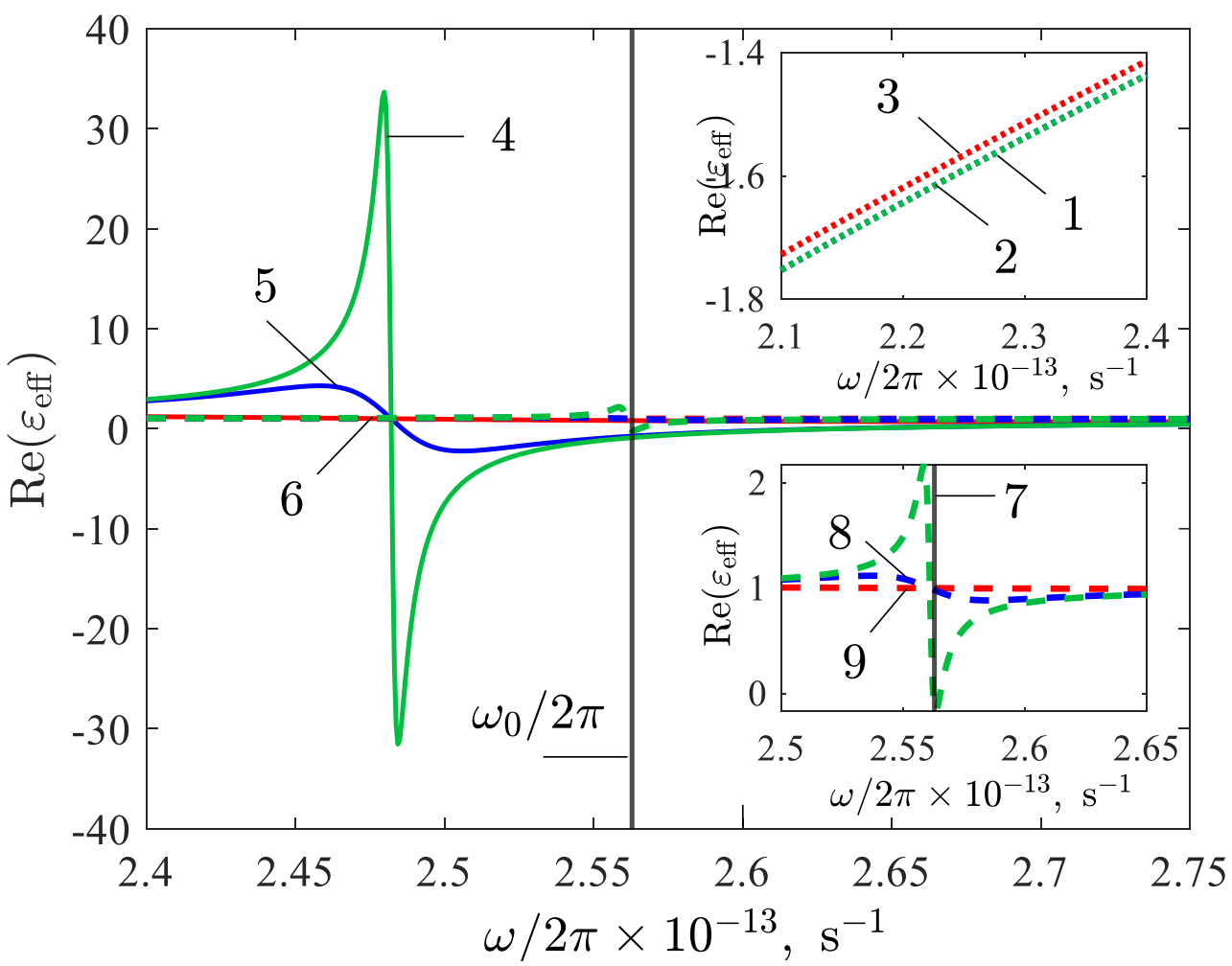

Fig. 10. Dependence of a real part of effective dielectric function $\varepsilon_{\text {eff }}$ on the frequency the CNT array, based on the arrays geometrical model.

\section{Discussion of results}

From the calculation results shown in Fig. 1, it follows that in the case of the multicascade mechanism of radiation generation at $\alpha_{R}=2$ the length of the unsteady mode region is equal to $0 \leq t \leq 0.3 \times 10^{-11} \mathrm{~s}$. The solution of quantity $\not 6(t, \bar{\varepsilon})$ in the length of steady mode region $t \geq 0.3 \times 10^{-11} \mathrm{~s}$ has a form of plateau, i.e. the solution doesn't depend on time. From the calculation results shown in the inset of Fig. 1, a good match of the calculation results at moment $t=10^{-11} \mathrm{~s}$ at different temperatures $T=150,300,600 \mathrm{~K}$ with analytical solution (3.6) follows. From the calculation results shown in Fig. 2, it follows that at $\alpha_{R}=4$ the length of the unsteady mode region is equal to $0 \leq t \leq 0.8 \times 10^{-11} \mathrm{~s}$. The solution of quantity $\left.\not 6 t, \bar{\varepsilon}\right)$ in the region of the length of steady mode region) $t \geq 0.8 \times 10^{-11} \mathrm{~s}$ has a ridge-shaped form.

Fig. 3 shows the results of calculations that correspond to the mechanism of radiation generation, when the current that is obtained in the mechanism of radiation generation during the transition from the initial state to the final state is associated with stimulated absorption. We will 
assume that the current has a dependence, as defined in (2.7). From the calculation results shown in Fig. 3, it follows that at $\alpha_{R}=2$ the length of the unsteady mode region is equal to $0 \leq t \leq 0.3 \times 10^{-11} \mathrm{~s}$ as in the case shown in Fig. 1 . The solution of quantity $t \geq 0.3 \times 10^{-11} \mathrm{~s}$ has a form of plateau, i.e. the solution doesn't depend on time. From the calculation results shown in Fig. 4, it follows that at $\alpha_{R}=4$ the length of the unsteady mode region is equal to $0 \leq t \leq 0.8 \times 10^{-11} \mathrm{~s}$. The solution of quantity $\rho(t, \bar{\varepsilon})$ in the region of the length of steady mode region $t \geq 0.8 \times 10^{-11} \mathrm{~s}$ has a ridge-shaped form. From the calculation results shown in the inset of Fig. 3, it follows that a quantity $\rho$ at initial moment of time $t=0.004 \times 10^{-11}$ s (curve 2) reduces because of a transition from the initial state $\left|k_{1}\right\rangle$ to the final state $\left|k_{0}\right\rangle$ due to the stimulated absorption of infrared radiation).

At wave length $\lambda=65 \mu \mathrm{m}\left(\omega \approx \Omega=2.7 \times 10^{13} \mathrm{~s}^{-1}\right)$ terahertz radiation meets requirement $\omega /(2 \pi) \approx 0.43 \times 10^{13} \mathrm{~s}^{-1}$. From the calculation results shown in Fig. 5-10, it follows that terahertz radiation is far from maximum point $\omega_{0}=1.6 \times 10^{14} \mathrm{~s}^{-1}$ of function $\operatorname{Im} \varepsilon_{\text {eff }}(\omega)$. Discussed in the work volume ratios on the basis EMA, the Maxwell-Garnett approximation and the arrays geometrical model are equal to $f=1.44 \times 10^{-3} ; 3.9 \times 10^{-5} ; 1.44 \times 10^{-6}$. It corresponds to average distances between nanotubes $30 \mathrm{~nm}, 100 \mathrm{~nm}$, 300nm on the basis EMA, the Maxwell-Garnett approximation. In the calculations, where the arrays geometrical model is used, the tetragonal lattice parameters are chosen as follows: when the distance between nanotubes is $\Delta x \times \Delta y \times \Delta z=20 \times 20 \times 67.5 \mathrm{~nm}$, the volume fraction is $f=1.44 \times 10^{-3}$, the geometrical factor is $B_{S}=0.44$; when the distance between nanotubes is $\Delta x \times \Delta y \times \Delta z=71 \times 71 \times 198.4 \mathrm{~nm}$, the volume fraction is $f=3.9 \times 10^{-5}$, geometrical factor $B_{s}=0.53$; when the distance between nanotubes is, the volume fraction is $\Delta x \times \Delta y \times \Delta z=300 \times 300 \times 300 \mathrm{~nm}$, the volume fraction is $f=1.44 \times 10^{-6}$, geometrical factor $B_{s}=1 / 3$. Obtained in the work parameters of the medium consisting of the extended nanoparticles, based on the arrays geometrical model are interesting from the position of the short terahertz impulses generation [69-71]

\section{Conclusion}


The results obtained in this paper show that in the case of the multicascade mechanism of radiation generation in the medium, based on noninteracting parallel aligned CNTs can be used as a non-linear amplification region of radiation. The process of the terahertz amplification with a QC-laser in the medium, based on the armchair-edge nanoribbons and the zigzag nanotubes with the metallic conductivity is theoretically studied and numerically simulated in the paper. The quantity of injection current in the nanopaticle points is rated. The task is generalized for analogous mediums in the case where radiation current can be generated with the help of the process of infrared radiation stimulated absorption, for example, using the radiation of a $\mathrm{CO}_{2}$ laser.

In the case of the multi cascade mechanism of radiation generation in a continuous wave mode the field is equal to $|\bar{E}|=2.4 \times 10^{5} \mathrm{~V} / \mathrm{m}$ (see 3.7), where $T_{2}=3 \times 10^{-12} \mathrm{~s}$. The quantity of the field intensity is the result of the stream (3.7) quantity $\rho$ conservation in a continuous wave mode (consequence of the charge conservation law). If concentration of nanotubes is equal to $n_{0}=10^{10} \mathrm{c} \mathrm{m}^{-2}=10^{14} \mathrm{~m}^{-2}$ (the distance between nanotubes is $100 \mathrm{~nm}$, then the output power per unit area will be about $Q \sim n_{0} k_{0} \mathrm{~h} \Omega J_{k_{0}} / e \approx 860 \mathrm{~W} / \mathrm{cm}^{2}$, where $J_{k_{0}}=8 \times 10^{-6} \mathrm{~A}$, $k_{0}=6, \bar{\varepsilon}_{\kappa_{0}}=k_{0} \mathrm{~h} \Omega=0.11 \mathrm{eV}$.

In the task of terahertz radiation generation with the use of a QC-laser in the medium, based on the the armchair-edge nanoribbons and the zigzag nanotubes with the metallic conductivity, the value for transition rate $W^{(a)} \approx 9.26 \times 10^{13} \mathrm{~s}^{-1}$ with stimulated absorption of radiation of a $\mathrm{CO}_{2}$ laser (it corresponds to the injection current $J_{k_{0}}=-J_{k_{1}} \approx 1.48 \times 10^{-5} \mathrm{~A}$ in nanoparticles) has been obtained. In the discussed case the current quantity is 1.85 times larger then in the case of the multicascade mechanism of radiation generation. Field intensity in a continuous wave mode the field is equal to $|\bar{E}|=3.26 \times 10^{5} \mathrm{~V} / \mathrm{m}$.

From given in the paper numerical results for the effective dielectric function it follows, that for the terahertz radiation with wave length $\lambda=65 \mu \mathrm{m}\left(\omega \approx \Omega=2.7 \times 10^{13} \mathrm{~s}^{-1}\right)$ condition $\omega<<\omega_{0}$, where $\omega_{0}=1.6 \times 10^{14} \mathrm{~s}^{-1}$, is executed). It means, that frequency of terahertz radiation is far from maximum point $\omega_{0}$ of function $\operatorname{Im} \varepsilon_{\text {eff }}(\omega)$. In this region of frequency the quantity of imaginary and real parts of the effective dielectric function has reasonable values.

\section{Acknowledgment}


The authors of the paper would like to thank for the support from National Research Nuclear University MEPhI in the framework of the Russian Academic Excellence Project (contract No. 02.a03.21.0005. 27.08.2013).

\section{Declarations}

\section{Funding}

No funding.

\section{Conflicts of interest}

No conflict of interest.

\section{Availability of data and material}

The data that supports the findings of this study are available within the article.

\section{Code availability}

Not applicable.

\section{Appendix}

In terms of electrodynamics, the discrete structure of massive amount of dispersive particles is equal to a continuous dielectric material, characterized by the effective dielectric function (magnetic properties of particles are neglected) (See [63])

$$
\hat{\varepsilon}_{\mathrm{eff}}=\varepsilon_{2}\left[1+\frac{4 \pi}{\Omega} \hat{g}\left(1+\frac{1}{\Omega} \hat{\delta} \cdot \hat{g}\right)^{-1}\right],
$$

hereinafter, the axis of nanotube is directed along the $\mathrm{z}$ axis, where $\Omega=\Delta x \Delta y \Delta z, \Delta x, \Delta y, \Delta z$ are the distances between nanotube centers along the axes $x, y$ and $z$, correspondently; the production of operators $\hat{\delta} \cdot \hat{g}$ paper is equal to $\hat{\delta} \hat{g}=\delta_{i i} \cdot g_{i i}$, where $i=1,2,3$,

$$
\begin{aligned}
& \delta_{11}=-4 \pi \delta_{x x} / 3, \quad \delta_{22}=-4 \pi \delta_{y y} / 3, \quad \delta_{33}=-4 \pi \delta_{z z} / 3, \\
& \delta_{x x}=3 B_{p}, \quad \delta_{y y}=3 B_{p}, \quad \delta_{z z}=3 B_{s},
\end{aligned}
$$

Where $B_{s}$ and $B_{p}$ are the geometrical factors, such, that the polarization is aligned along ( $S_{-}$ polarization) or perpendicular ( $p$ - polarization) to the nanotube's axis). 
In the case where the tensor of dielectric permittivity of ellipsoid is diagonal and main direction of this tensor coincide with the main axes of ellipsoid, the matrix $g_{i k}$ is also diagonal ( $\left.g_{i j}=0, i \neq j\right)$ and have form (see [63])

$$
g_{i i}=\frac{a b c}{3} \frac{\left(\varepsilon_{i i}-\varepsilon_{2}\right)}{\varepsilon_{2}+n_{i}\left(\varepsilon_{i i}-\varepsilon_{2}\right)}, \quad n_{i}=\frac{a b c}{2} J_{i} \text {, }
$$

where $J_{1}=J_{100}, \quad J_{2}=J_{010}, \quad J_{3}=J_{001}, n_{i}$ - is the depolarization factor [50].

$$
\begin{aligned}
& J_{i}=\int_{0}^{\infty} \frac{d \xi}{\left(\xi+\eta_{i}^{2}\right) \sqrt{R(\xi)}}, \quad R(\xi)=\left(a^{2}+\xi\right)\left(b^{2}+\xi\right)\left(c^{2}+\xi\right), \\
& \eta_{1}=a, \quad \eta_{2}=b, \quad \eta_{3}=c .
\end{aligned}
$$

From (4.1) and (A3) it follows that $g_{i i}$ coincide with the polarizability $\alpha_{i}$

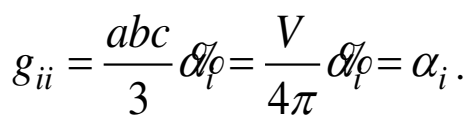

From (A1), taking into account (A2) and (A3), we obtain the expression for the effective dielectric function

$$
\hat{\varepsilon}_{\mathrm{eff}}=\varepsilon_{2}\left[1+\frac{f \mathscr{O}_{\chi}}{1-f B_{\chi} \mathscr{Q}_{\chi}}\right]
$$

Where $f=4 \pi a b c /(3 \Omega)$ is the volume fraction; $B_{\chi}$ is a geometrical factor, $\chi=s, p ; B_{s}$ is such a geometrical factor, that the polarization is aligned along (s-polarization), $B_{p}$ is such a geometrical factor, that the polarization is perpendicular to the nanotube's axis (p-polarization)). In tetragonal lattice $\Delta x=\Delta y, \Delta z / \Delta x=\beta$ the geometrical factors are equal to the ones from [63]; thus, it follows that

$$
\begin{aligned}
& B_{s}=\frac{\delta_{z z}}{3}=\frac{1}{4 \pi}\left[\left(18-\frac{10}{\beta^{2}}\right) \arctan \frac{Q}{\beta^{2}}+30 \frac{1-\beta^{2}}{\beta^{4}}\left(2 \arctan Q-\ln \frac{1+Q}{1-Q}\right)\right], \\
& B_{p}=\frac{\delta_{x x}}{3}=\frac{\delta_{y y}}{3}=\frac{1}{4 \pi}\left[\left(18+\frac{20}{\beta^{2}}-\frac{30}{\beta^{4}}\right) \arctan Q+15 \frac{1-\beta^{2}}{\beta^{4}} \ln \frac{1+Q}{1-Q}\right],
\end{aligned}
$$

where $Q=\beta / \sqrt{2+\beta^{2}}$. 


\section{References}

1. C. Gmachl, F. Capasso, D.L. Sivco, A.Y. Cho: Recent progress in quantum cascade lasers and applications. Rep. Prog. Phys. 64, 1533-1601 (2001). https://doi.org/10.1088/0034-4885/64/11/204

2. R.F. Kazarinov, R.A. Suris: Possibility of amplification of electromagnetic waves in a semiconductor with a superlattice. Fiz. Tekh. Poluprov. 5, 797-800 (1971).

3. J. Faist, E. Capasso, D.L. Sivico, C. Sirtori, A.L. Hutchinson, A.Y. Cho: Quantum cascade laser. Science 264, 553-556 (1994). https://doi.org/10.1126/science.264.5158.553

4. F. Capasso, C. Gmachl, D.L. Sivco, A.Y. Cho: Quantum cascade lasers. Phys. World 12, 27-34 (1999).

5. D. Zhang, B.H. Yang, R.Q. Yang, C.-H. Lin, S.J. Murry, S.S. Pei: Mid-infrared interband cascade lasers with quantum efficiencies > 200\%. Appl. Phys. Lett. 72(18), 2220-2222. https://doi.org/10.1063/1.121265

6. J. Faist, F. Capasso, C. Sirtori, D.L. Sivco, A.L. Hutchinson, and A.Y. Cho, "Continuous wave operation of a vertical transition quantum cascade laser above T=80 K", Applied Physics Letters 67, 3057-3059 (1995). https://doi.org/10.1063/1.114863

7. J. Faist, F. Capasso, C. Sirtori, D.L. Sivco, A.L. Hutchinson, and A.Y. Cho, "Vertical transition quantum cascade laser with Bragg confined excited state", Applied Physics Letters 66, 538-540 (1995). https://doi.org/10.1063/1.114005

8. J. Faist, F. Capasso, C. Sirtori, D.L. Sivco, J.N. Baillargeon, A.L. Hutchinson, S.-N. G. Chu, and A.Y. Cho, "High power mid-infrared $(\lambda \sim 5 \mu \mathrm{m})$ quantum cascade lasers operating above room temperature", Applied Physics Letters 68, 3680-3682 (1996). https://doi.org/10.1063/1.115741

9. Y. Bai, N. Bandyopadhyay, S. Tsao, S. Slivken, and M. Razeghi, Appl. Phys. Lett. 98, 181102 (2011). https://doi.org/10.1063/1.3586773

10. O. Cathabard, R. Teissier, J. Devenson, J. C. Moreno, and A. N. Baranov, Appl. Phys. Lett. 96, 141110 (2010) https://doi.org/10.1063/1.3385778

11. G. Scalari, D. Turc`inkova, J. Lloyd-Hughes, M. I. Amanti, M. Fischer, M. Beck, and J. Faist, Appl. Phys. Lett. 97, 081110 (2010).

https://doi.org/10.1063/1.3481698 
12. R. Colombelli, F. Capasso, C. Gmachl, A.L. Hutchinson, D.L. Sivco, A. Tredicucci, M.C. Wanke, A.M. Sergent, A.Y. Cho: Far-infrared surface-plasmon quantum-cascade lasers at $21.5 \mathrm{~lm}$ and $24 \mathrm{~lm}$ wavelengths. Appl. Phys. Lett. 78, 2620-2622 (2001). https://doi.org/10.1063/1.1367304

13. A.K. Wojcik, P. Malara, R. Blanchard, T.S. Mansuripur, F. Capasso, A. Belyanin: Generation of picosecond pulses and frequency combs in actively mode locked external ring cavity quantum cascade lasers. Appl. Phys. Lett. 103, 231102 (2013). https://doi.org/10.1063/1.4838275

14. S. Barbieri, M. Ravaro, P. Gellie, G. Santarelli, C. Manquest, C. Sirtori, S.P. Knahna, E.H. Linfield, A.G. Davies: Coherent sampling of active mode-locked terahertz quantum cascade lasers and frequency synthesis. Nat. Photonics 5, 306313 (2011). https://doi.org/10.1038/nphoton.2011.49

15. J. Freeman, J. Maysonnave, N. Jukam, P. Cavali, K. Maussang, H. Beere, D. Ritchie, J. Mangeney, S. Dhillon, J. Tignon: Direct intensity sampling of a modelocked terahertz quantum cascade laser. Appl. Phys. Lett. 101, 181115 (2012). https://doi.org/10.1063/1.4765660

16. C. Jirauschek and T. Kubis, Appl. Phys. Rev., V. 1, 011307-51 (2014) https://doi.org/10.1063/1.4863665

17. Q. Y. Lu, N. Bandyopadhyay, S. Slivken, Y. Bai, and M. Razeghi, Opt. Express 21, 968 (2013). https://doi.org/10.1364/OE.21.000968

18. K. Vijayraghavan, Y. Jiang, M. Jang, A. Jiang, K. Choutagunta, A. Vizbaras, F. Demmerle, G. Boehm, M. C. Amann, and M. A. Belkin, Nat. Commun. 4, 2021 (2013). http://doi.org/10.1038/ncomms3021

19. C. Jirauschek, A. Matyas, P. Lugli, and M.-C. Amann, Opt. Express 21, 6180 (2013). https://doi.org/10.1364/OE.21.006180

20. S. Fathololoumi, E. Dupont, C. Chan, Z. Wasilewski, S. Laframboise, D. Ban, A. Matyas, C. Jirauschek, Q. Hu, and H. Liu, Opt. Express 20, 3866 (2012). https://doi.org/10.1364/OE.20.003866

21. M. A. Belkin, J. A. Fan, S. Hormoz, F. Capasso, S. P. Khanna, M. Lachab, A. G. Davies, and E. H. Linfield, Opt. Express 16, 3242 (2008).

https://doi.org/10.1364/OE.16.003242 
22. K. Vijayraghavan, R. W. Adams, A. Vizbaras, M. Jang, C. Grasse, G. Boehm, M. C. Amann, and M. A. Belkin, Appl. Phys. Lett. 100, 251104 (2012). https://doi.org/10.1063/1.4729042

23. O. Demichel, L. Mahler, T. Losco, C. Mauro, R. Green, A. Tredicucci, J. Xu, F. Beltram, H. E. Beere, D. A. Ritchie, and V. Tamosinuas, Opt. Express 14, 5335 (2006). https://doi.org/10.1364/OE.14.005335

24. Y. Chassagneux, R. Colombelli, W. Maineult, S. Barbieri, H. E. Beere, D. A. Ritchie, S. P. Khanna, E. H. Linfield, and A. G. Davies, Nature 457, 174 (2009). https://doi.org/10.1038/nature07636

25. R. Colombelli, K. Srinivasan, M. Troccoli, O. Painter, C. F. Gmachl, D. M. Tennant, A. M. Sergent, D. L. Sivco, A. Y. Cho, and F. Capasso, Science 302, 1374 (2003). https://doi.org/10.1126/science.1090561

26. M. Schubert and F. Rana, IEEE J. Quantum Electron. 42, 257 (2006). https://doi.org/10.1109/JQE.2005.863138

27. A. Taflove and S. C. Hagness, The Finite-Difference Time-Domain Method (Artech House, Boston, 2000)

28. M.I. Katsnelson, Just Add Water, Science, 329 (2010) 1157-1158. https://doi.org/10.1126/science.1195392

29. S. V Morozov, K.S. Novoselov, A.K. Geim, Electron transport in graphene, Physics-Uspekhi. 51 (2008) 744-748. https://doi.org/10.1070/pu2008v051n07abeh006575

30. R. Saito, M. Fujita, G. Dresselhaus, M.S. Dresselhaus, Electronic structure of graphene tubules based on C60, Phys. Rev. B. 46 (1992) 1804-1811. https://doi.org/10.1103/PhysRevB.46.1804

31. A. Onipko and L. Malysheva, Electron Spectrum of Graphene Macromolecule Revisited, Phys. Status Solidi 255 (2017) 1700248.

https://doi.org/10.1002/pssb.201700248

32. L.I. Malysheva, A.I. Onipko, Quantum Conductance of Achiral Graphene Ribbons and Carbon Tubes, J. Exp. Theor. Phys. 108 (2009) 126-131. https://doi.org/10.1134/S1063776109010166

33. N. R. Sadykov, S.E. Jolnirov, Physica E: Low-dimensional Systems and Nanostructures. 2021. V. 128. P. 114574 (1-10).

https://doi.org/10.1016/j.physe.2020.114574 
34. F.-L. Yan, J.-C. Zhang, Z.-W. Jia, N. Zhuo, S.-Q. Zhai, S.-M. Liu, F.-Q. Liu, and Z.-G. Wang, AIP Advances, V. 6, 035022 (1-6) (2016).

https://doi.org/10.1063/1.4945383

35. Sadykov N. R., Muratov E.T., Pilipenko I. A., Aporoski A. V., Physica E: Low-dimensional Systems and Nanostructures. 2020. V. 120. P. 114071 (1-11). https://doi.org/10.1016/j.physe.2020.114071

36. N. R. Sadykov, A.V. Aporoski, D.A. Peshkov, Terahertz radiation generation process in the medium based on the array of the noninteracting nanotubes, Opt. Quantum Electron. 48 (2016). https://10.1007/s11082-016-0625-8

37. N. R. Sadykov, A. V Aporoski, Generation of microwave and terahertz radiation in a medium of nanoparticles, Opt. Quantum Electron. 51 (2019) 96. https://10.1007/s11082-019-1811-2

38. N.R. Sadykov, A. V. Aporoski, Majorana fermion wavefunctions in carbon nanotubes and carbynes, Int. J. Mod. Phys. B. 31 (2017) 1750017. https://10.1142/S0217979217500175

39. N.R. Sadykov, N.A. Skorkin: Quantum Approach to the Description of Amplification of Radiation From an Array of Nanotubes. Tech. Phys. 58, 625-629 (2013). https://10.1134/S1063784213050186

40. N.R. Sadykov: Wave functions and eigenvalues of charge carriers in a nanotube in a neighborhood of the dirac point in the presence of a longitudinal electric field. Theor. Math. Phys. 180(3), 1073-1085 (2014). https://10.4213/tmf8642

41. R. W. Boyd, Nonlinear Optics (Academic Press, San Diego, 2003)

42. K. Sasaki, K. Wakabayshi, T. Enoki, Electron wave function in armchair graphene nanoribbons, J. Phys. Soc. Jpn. 80 (1-7) (2011), 044710, https://doi.org/10.1143/JPSJ.80.044710

43. K. Wakabayashi, K.-I. Sasaki, T. Nakanishi, T. Enoki, Electronic states of graphene nanoribbons and analytical solutions, Sci. Technol. Adv. Mater. (2010), 054504. https://doi.org/10.1088/1468-6996/11/5/05450

44. L. D. Landau and E. M. Lifshitz, Course of Theoretical Physics, Vol. 3: Quantum Mechanics: Non-Relativistic Theory, 4th ed. (Oxford Univ. Press, Oxford, 1980; Nauka, Moscow, 1989).

45. V. B. Berestetskii, E. M. Lifshitz, L. P. Pitaevskii (1971). Relativistic Quantum Theory. Vol. 4 (1st ed.). Pergamon Press. ISBN 978-0-08-017175-3.

46. R. Landauer, Phil. Mag. 21, 863 (1972) 
47. M. Buttiker, Phys. Rev. B 46, 12485 (1992). https://doi.org/10.1103/

48. J. H. Davies, The Physics of Low-Dimensional Semiconductors (Cambridge University Press, Cambridge, 1997. https://doi.org/10.1017/CBO9780511819070

49. G. N. Grachev , A. A. Zemlyanov , A. G. Ponomarenko, V. N. Tishchenko, Yu. E. Geints, A. M. Kabanov , A. A. Pavlov, d, Al. A. Pavlov, d, V. A. Pogodaev, P. A. Pinaev, A. L.

Smirnov , and P. A. Statsenko, Atmospheric and Oceanic Optics, 2014, Vol. 27, No. 2, pp. 115 122. https://doi.org/10.1134/S1024856014020067

50. L. D. Landau and E. M. Lifshitz, Course of Theoretical Physics, vol. 8. Electrodynamics of Continuous Media,. (Oxford University Press, 1960).

51. W. Lü, J. Dong, Z.-Y. Li, Optical properties of aligned carbon nanotube systems studied by the effective-medium approximation method, Phys. Rev. B 63 (2000), 033401. https://doi.org/10.1103/PhysRevB.63.033401

52. J. C. Maxwell-Garnett, Philos. Trans. R. Soc. 203, 385 (1904).

www.doi.org/10.1098/rsta.1904.0024

53. D. M. Wood and N. W. Ashcroft, Philos. Mag. 35, 269 (1977). https://doi.org/10.1080/14786437708237052

54. S. Datta, C. T. Chan, K. M. Ho, and C. M. Soukoulis, Effective dielectric constant of periodic composite structures, Phys. Rev. B 48, 14936-14943, (1993).

https://doi.org/10.1103/PhysRevB.48.14936

55. R. Tao, Z. Chen, and P. Sheng, Phys. Rev. B 41, 2417 (1990).

https://doi.org/10.1103/physrevb.41.2417

56. D. A. G. Bruggeman, Ann. Phys. (Leipzig) 24, 636 (1935).

https://doi.org/10.1002/andp.19354160705

57. Landauer, in Electrical Transport and Optical Properties in Inhomogeneous Media, edited by J. C. Garland and D. B. Tanner, AIP Conf. Proc. No. 40 (Woodbury, New York, 1978), p. 2

58. G. A. Niklasson and C. G. Granqvist, J. Appl. Phys. 55, 3382 (1984).

https://doi.org/10.1063/1.333386

59. F. Brouers, J. Phys. C 19, 7183 (1986); S. Berthier, Ann. Phys. (Paris) 13, 503 (1988).

60. S.S. Savinskii, N. V. Khokhryakov, Characteristic features of the $\pi$-electron states of carbon nanotubes, J. Exp. Theor. Phys. 84 (1997) 1131-1137. https://doi.org/10.1134/1.558250.53

61. J.C.M. Garnett, Colours in Metal Glasses and in Metallic Films, Philos. Trans. R. Soc. A Math. Phys. Eng. Sci. 203 (1904) 385-420. https://doi.org/10.1098/rsta.1904.0024

62. V.A. Markel, Introduction to the Maxwell Garnett approximation: tutorial, J. Opt. Soc. Am. A. 33 (2016) 1244. https://doi.org/10.1364/JOSAA.33.001244 
63. N. A. Khizhnyak, Integral equations of macroscopic electrodynamics, Naukova Dumka, Kiev, 1986.

64. F.J. García-Vidal, J.M. Pitarke, J.B. Pendry, Effective Medium Theory of the Optical Properties of Aligned Carbon Nanotubes, Phys. Rev. Lett. 78 (1997) 4289-4292. https://doi.org/10.1103/PhysRevLett.78.4289

65. S. Tasaki, K. Maekawa, and T. Yamabe, Phys. Rev. B 57, 9301 (1998). https://doi.org/10.1103/PhysRevB.57.9301

66. Akhlesh Lakhtakia, Gregory Ya. Slepyan, Sergey A. Maksimenko, Anton V. Gusakov, Oleg M. Yevtushenko, Effective medium theory of the microwave and the infrared properties of composites with carbon nanotube inclusions, Carbon 36, 1833-1839, (1998),

https://doi.org/10.1016/S0008-6223(98)00155-9

67. G. Ya. Slepyan, S. A. Maksimenko, V. P. Kalosha, J. Herrmann, I. L. Krestnikov and Zh. I. Alferov, D. Bimberg, Phys. Rev. B 59, 12275-12278 (1999).

https://doi.org/10.1103/PhysRevB.59.12275

68. G.Y. Slepyan, M.V. Shuba, S.A. Maksimenko, C. Thomsen, A. Lakhtakia: Terahertz conductivity peak in composite materials containing carbon nanotubes: theory and interpretation of experiment. Phys. Rev. B 81, 205423 (2010). https://doi.org/10.1103/PhysRevB.81.205423

69. M.B. Belonenko, E.V. Demushkina, N.G. Lebedev, J. Rus. Las. Res. 27, 457 (2006). https://doi.org/10.1007/s10946-006-0027-7

70. A.V. Zhukov, R. Bouffanais, B.A. Malomed, H. Leblond, D. Mihalache, E.G. Fedorov, N.N. Rosanov, M.B. Belonenko, Phys. Rev. A. 94, 053823 (2016).

https://doi.org/10.1103/PhysRevA.94.053823

71. N. R. Sadykov, D. A. Peshkov A. V. Aporoski, M. B. Belonenko. Decay, Amplification and Absorption of Initial Terahertz Pulse in Resonant Two-Level Medium Based on Non-Interacting Array of Zigzag Nanotubes and Armchair Nanoribbons, Int. J. Mod. Phys. B, 2020, 2050202 (125). https://doi.org/10.1142/S0217979220502021 